\title{
A REDUCTION PRINCIPLE FOR THE CRITICAL VALUES OF RANDOM SPHERICAL HARMONICS
}

\author{
VALENTINA CAMMAROTA AND DOMENICO MARINUCCI
}

\begin{abstract}
We study here the random fluctuations in the number of critical points with values in an interval $I \subset \mathbb{R}$ for Gaussian spherical eigenfunctions $\left\{f_{\ell}\right\}$, in the high energy regime where $\ell \rightarrow \infty$. We show that these fluctuations are asymptotically equivalent to the centred $L^{2}$-norm of $\left\{f_{\ell}\right\}$ times the integral of a (simple and fully explicit) function over the interval under consideration. We discuss also the relationships between these results and the asymptotic behaviour of other geometric functionals on the excursion sets of random spherical harmonics.
\end{abstract}

- AMS Classification: 60G60, 62M15, 53C65, 42C10, 33C55.

- Keywords and Phrases: Reduction Principle, Critical Points, Wiener-Chaos Expansion, Spherical Harmonics, Quantitative Central Limit Theorem, Berry's Cancellation Phenomenon

\section{Introduction and Main Result}

1.1. The asymptotic geometry of random spherical harmonics. It is well-known that the eigenvalues $\lambda$ of the Laplace equation $\Delta_{\mathbb{S}^{2}} f+\lambda f=0$ on the two-dimensional sphere $\mathbb{S}^{2}$, are of the form $\lambda=\lambda_{\ell}=\ell(\ell+1)$ for some integer $\ell \geq 1$. For any given eigenvalue $\lambda_{\ell}$, the corresponding eigenspace is the $(2 \ell+1)$-dimensional space of spherical harmonics of degree $\ell$; we can choose an arbitrary $L^{2}$-orthonormal basis $\left\{Y_{\ell m}(.)\right\}_{m=-\ell, \ldots, \ell}$, and consider random eigenfunctions of the form

$$
f_{\ell}(x)=\frac{\sqrt{4 \pi}}{\sqrt{2 \ell+1}} \sum_{m=-\ell}^{\ell} a_{\ell m} Y_{\ell m}(x),
$$

where the coefficients $\left\{a_{\ell m}\right\}$ are complex-valued Gaussian variables, such that for $m \neq 0, \operatorname{Re}\left(a_{\ell m}\right), \operatorname{Im}\left(a_{\ell m}\right)$ are zero-mean, independent Gaussian variables with variance $\frac{1}{2}$, while $a_{\ell 0}$ follows a standard Gaussian distribution. The random fields $\left\{f_{\ell}(x), x \in \mathbb{S}^{2}\right\}$ are isotropic, meaning that the probability laws of $f_{\ell}(\cdot)$ and $f_{\ell}^{g}(\cdot):=f_{\ell}(g \cdot)$ are the same for any rotation $g \in S O(3)$. Also, $f_{\ell}$ are centred Gaussian, and from the addition theorem for spherical harmonics (see i.e., 222, eq. (3.42)) the covariance function is given by,

$$
\mathbb{E}\left[f_{\ell}(x) f_{\ell}(y)\right]=P_{\ell}\left(\cos \theta_{x y}\right), \quad \theta_{x y}:=d_{\mathbb{S}^{2}}(x, y),
$$

where $P_{\ell}$ are the usual Legendre polynomials, defined by

$$
P_{\ell}(t)=\frac{1}{2^{\ell} \ell !} \frac{d^{\ell}}{d t^{\ell}}\left(t^{2}-1\right)^{\ell}, \quad t \in[-1,1], \quad \ell \in \mathbb{N},
$$

whereas $d_{\mathbb{S}^{2}}(x, y)$ is the usual spherical geodesic distance between $x$ and $y$, i.e.,

$$
d_{\mathbb{S}^{2}}(x, y)=\arccos (\langle x, y\rangle) \text {, where }\langle x, y\rangle=\cos \theta_{x} \cos \theta_{y}+\sin \theta_{x} \sin \theta_{y} \cos \left(\varphi_{x}-\varphi_{y}\right),
$$

and $\left(\theta_{x}, \varphi_{x}\right),\left(\theta_{y}, \varphi_{y}\right)$ are the spherical coordinates of $x$ and $y$ respectively, $\theta_{a} \in[0, \pi], \varphi_{a} \in[0,2 \pi), a=x, y$.

A number of issues on the geometry of random spherical harmonics has been scrutinized recently, including the number of nodal domains 229, the length of nodal lines [37, 25], the excursion area and the defect 27, 24, critical values and the Euler-Poincaré characteristic [7, 8, 9], 10, mass equidistribution 19, critical radius 17]; these and/or other geometric features for random eigenfunctions on other compact manifolds such as the torus (arithmetic random waves) or on the plane (Berry's random waves model, [5]) have also been intensively studied, see i.e. 44, 14, 20, 23, 231, 32 for the fluctuations of nodal lengths, 6] for the number of nodal domains, 18 for the analysis of mass equidistributions and [34, 35] for nodal intersections, to list some of the most recent contributions; a review is given in [33].

Department of Statistics, Sapienza University of Rome

Department of Mathematics, University of Rome Tor Vergata 
In particular, consider the excursion sets $A_{u}\left(f_{\ell} ; \mathbb{S}^{2}\right)$, defined for $u \in \mathbb{R}$ by

$$
A_{u}\left(f_{\ell} ; \mathbb{S}^{2}\right):=\left\{x \in \mathbb{S}^{2}: f_{\ell}(x) \geq u\right\} ;
$$

their geometry can be characterized by the behaviour of the Lipschitz-Killing curvatures $\mathcal{L}_{k}, k=0,1,2,($ see i.e., [1, [2]), which in dimension two correspond respectively to the Euler-Poincaré characteristic $\mathcal{L}_{0}$, (half) the boundary length $\mathcal{L}_{1}$, and the excursion area $\mathcal{L}_{2}$; the asymptotic behaviour (in the high-energy regime $\ell \rightarrow \infty$ ) of these three quantities is now fully understood, and indeed we have (see [9])

$$
\begin{aligned}
& \mathcal{L}_{0}\left(A_{u}\left(f_{\ell} ; \mathbb{S}^{2}\right)\right)-\mathbb{E}\left[\mathcal{L}_{0}\left(A_{u}\left(f_{\ell} ; \mathbb{S}^{2}\right)\right)\right]=\frac{1}{2}\left\{\frac{\lambda_{\ell}}{2}\right\}\left[H_{2}(u) H_{1}(u) \phi(u)\right] \frac{1}{2 \pi} \int_{\mathbb{S}^{2}} H_{2}\left(f_{\ell}(x)\right) d x+R_{0}(\ell), \\
& \mathcal{L}_{1}\left(A_{u}\left(f_{\ell} ; \mathbb{S}^{2}\right)\right)-\mathbb{E}\left[\mathcal{L}_{1}\left(A_{u}\left(f_{\ell} ; \mathbb{S}^{2}\right)\right)\right]=\frac{1}{2}\left\{\frac{\lambda_{\ell}}{2}\right\}^{1 / 2} \sqrt{\frac{\pi}{8}}\left[H_{1}^{2}(u) \phi(u)\right] \int_{\mathbb{S}^{2}} H_{2}\left(f_{\ell}(x)\right) d x+R_{1}(\ell), \\
& \mathcal{L}_{2}\left(A_{u}\left(f_{\ell} ; \mathbb{S}^{2}\right)\right)-\mathbb{E}\left[\mathcal{L}_{2}\left(A_{u}\left(f_{\ell} ; \mathbb{S}^{2}\right)\right)\right]=\frac{1}{2}\left\{\frac{\lambda_{\ell}}{2}\right\}^{0}\left[H_{0}(u) H_{1}(u) \phi(u)\right] \int_{\mathbb{S}^{2}} H_{2}\left(f_{\ell}(x)\right) d x+R_{2}(\ell),
\end{aligned}
$$

where $\phi(u)=1 / \sqrt{2 \pi} \exp \left\{-u^{2} / 2\right\}$ denotes a standard Gaussian density, $H_{k}($.$) denotes Hermite polynomials$ $H_{k}(u)=(-1)^{k} \phi(u) \frac{d^{k} \phi(u)}{d u^{k}}$, and, for $k=0,1,2, R_{k}(\ell)$ are asymptotically negligible, meaning that

$$
\lim _{\ell \rightarrow \infty} \frac{\mathbb{E}\left[R_{k}^{2}(\ell)\right]}{\operatorname{Var}\left(\mathcal{L}_{k}\left(A_{u}\left(f_{\ell} ; \mathbb{S}^{2}\right)\right)\right)}=0
$$

The results given in (1.2)-(1.4) can be viewed as broadly analogues to the reduction principles established by 15 for the empirical processes of long-range dependent, stationary stochastic processes on $\mathbb{Z}$ : indeed these authors had shown that the empirical process of long range dependence sequences is asymptotically fully degenerate, being equivalent to a single random variable belonging to a Wiener chaos rescaled by a deterministic function depending only on the threshold value $u$. Likewise, the results reported in (1.2)-(1.4) entail some unexpected characterizations on the asymptotic behaviour of these geometric functionals, namely

(1) Any pair of them is asymptotically perfectly correlated at any non-zero threshold level, i.e.

$$
\lim _{\ell \rightarrow \infty} \operatorname{Corr}\left\{\mathcal{L}_{j}\left(A_{u_{1}}\left(f_{\ell} ; \mathbb{S}^{2}\right)\right), \mathcal{L}_{k}\left(A_{u_{2}}\left(f_{\ell} ; \mathbb{S}^{2}\right)\right)\right\}=1 \text {, for all } j, k=0,1,2, u_{1}, u_{2} \in \mathbb{R}, u_{1}, u_{2} \neq 0 ;
$$

in words, knowledge of the value of any of the three functionals allows to compute with asymptotically perfect precision the value of the other two functionals at any (non-zero) level

(2) It is possible to establish rather easily quantitative central limit theorems for each of them, by using standard tools; note indeed that, by Parseval's equality

$$
\int_{\mathbb{S}^{2}} H_{2}\left(f_{\ell}(x)\right) d x=\frac{4 \pi}{2 \ell+1} \sum_{m=-\ell}^{\ell}\left\{\left|a_{\ell m}\right|^{2}-\mathbb{E}\left|a_{\ell m}\right|^{2}\right\},
$$

i.e., each of the Lipschitz-Killing curvatures is a deterministic rescaling of a sum of centred, i.i.d. variables

(3) A phase transition occurs for $u=0$, where the leading terms disappear for all three functionals.

1.2. Statement of the main result. Our purpose in this paper is to provide a similar characterization for the behaviour of Gaussian spherical harmonics in terms of critical points. More precisely, let $I \subseteq \mathbb{R}$ be any interval in the real line; we are interested in the number of critical points with value in $I$ :

$$
\mathcal{N}_{\ell}^{c}(I)=\#\left\{x \in \mathbb{S}^{2}: f_{\ell}(x) \in I, \nabla f_{\ell}(x)=0\right\} .
$$

It has been shown in [7] that for every interval $I \subseteq \mathbb{R}$, as $\ell \rightarrow \infty$

$$
\mathbb{E}\left[\mathcal{N}_{\ell}^{c}(I)\right]=\frac{2}{\sqrt{3}} \ell(\ell+1) \int_{I} \pi^{c}(t) d t+O(1),
$$

where we have introduced the density function.

$$
\pi^{c}(t)=\frac{\sqrt{3}}{\sqrt{8 \pi}}\left(2 e^{-t^{2}}+t^{2}-1\right) e^{-\frac{t^{2}}{2}} .
$$


The constant in the $O(\cdot)$ term is universal; here (and later) by universality of the constant we mean that the integral of the error term on any interval $I$ is uniformly bounded by its value when $I=\mathbb{R}$, which is $\mathrm{O}(1)$. Also, we have

$$
\mathbb{E}\left[\mathcal{N}_{\ell}^{c}\right]:=\mathbb{E}\left[\mathcal{N}_{\ell}^{c}(\mathbb{R})\right]=\frac{2}{\sqrt{3}} \ell(\ell+1)+O(1)
$$

The investigation of the asymptotic variance of critical values is more challenging; we need first to establish some more notation, i.e. we shall define (see also [9])

$$
p_{3}^{c}(t)=\frac{1}{\sqrt{8 \pi}} e^{-\frac{3}{2} t^{2}}\left[2-6 t^{2}-e^{t^{2}}\left(1-4 t^{2}+t^{4}\right)\right], \quad \nu^{c}(I)=\left[\int_{I} p_{3}^{c}(t) d t\right]^{2}
$$

The following result was given in [7]: for every interval $I \subseteq \mathbb{R}$ as $\ell \rightarrow \infty$

$$
\operatorname{Var}\left(\mathcal{N}_{\ell}^{c}(I)\right)=\ell^{3} \nu^{c}(I)+O\left(\ell^{5 / 2}\right)
$$

again with a universal error bound in the $O(\cdot)$ term; similar results hold for the number of extrema and saddles.

Remark 1.1. It can be noted that for some intervals $I$ the leading constant $\nu^{c}(I)$ vanishes, and, accordingly, the order of magnitude of the variance is smaller than $\ell^{3}$; the most important among these cases is for $I=\mathbb{R}$ (corresponding to the total number of critical points), where we have [9], as $\ell \rightarrow \infty$

$$
\operatorname{Var}\left(\mathcal{N}_{\ell}^{c}\right)=\frac{1}{3^{3} \pi^{2}} \ell^{2} \log \ell+O\left(\ell^{2}\right)
$$

More generally, for the intervals such that the constant $\nu^{c}(I)$ vanishes, the variance of the number of critical points in $I$ has the following asymptotic behaviour: as $\ell \rightarrow \infty$

$$
\operatorname{Var}\left(\mathcal{N}_{\ell}^{c}(I)\right)=\left[\mu^{c}(I)\right]^{2} \ell^{2} \log \ell+O\left(\ell^{2}\right),
$$

where $\mu^{c}(I)=\int_{I} \mu^{c}(t) d t$, and the function $\mu^{c}(t)$ is given by

$$
\mu^{c}(t)=\frac{1}{2^{3} \pi} \sqrt{\frac{2}{\pi}}\left[\left(-2-36 t^{2}+38 t^{4}\right) e^{-t^{2}}+1+17 t^{2}-11 t^{4}+t^{6}\right] e^{-\frac{t^{2}}{2}} .
$$

Our main result in this paper is to establish a reduction principle for the number of critical points in the interval $I$, of similar nature as those given earlier in (1.2)-(1.4). In particular, following the same approach as given for other geometric functionals in many recent papers (see i.e., [23, [10, 31] and the references therein) we shall start by computing the $L^{2}(\Omega)$ expansion of critical points into Wiener Chaoses (the orthogonal spaces spanned by Hermite polynomials, see [30 for details), which will lead to lead to the $L^{2}(\Omega)$ expansion

$$
\mathcal{N}_{\ell}^{c}(I)=\sum_{q=0}^{\infty} \mathcal{N}_{\ell ; I}^{c}[q]
$$

indeed the rigorous justification of (1.8) and is one of the main technical challenges of this paper. The other main step is then to show that a single term dominates the $L^{2}(\Omega)$ expansion (after centering), then leading to the following

Theorem 1. As $\ell \rightarrow \infty$, we have that

$$
\begin{aligned}
\mathcal{N}_{\ell}^{c}(I)-\mathbb{E}\left[\mathcal{N}_{\ell}^{c}(I)\right] & =\frac{\ell}{2}\left[\int_{I} p_{3}^{c}(t) d t\right] \mathcal{N}_{\ell ; I}^{c}[2]+R_{\ell}(I) \\
& =\frac{\lambda_{\ell}}{2}\left[\int_{I} p_{3}^{c}(t) d t\right] \frac{1}{2 \pi} \int_{\mathbb{S}^{2}} H_{2}\left(f_{\ell}(x)\right) d x+R_{\ell}(I) \\
& =\frac{\ell+1}{2}\left[\int_{I} p_{3}^{c}(t) d t\right] \frac{2 \ell}{2 \ell+1} \sum_{m=-\ell}^{\ell}\left\{\left|a_{\ell m}\right|^{2}-1\right\}+R_{\ell}(I)
\end{aligned}
$$

where

uniformly over $I$.

$$
\operatorname{Var}\left(\mathcal{N}_{\ell ; I}^{c}[2]\right)=\ell^{3} \nu^{c}(I)+o\left(\ell^{3}\right), \mathbb{E}\left[R_{\ell}^{2}(I)\right]=o\left(\ell^{3}\right)
$$

Theorem 1 entails actually two different results, namely

a) the high frequency behaviour of the number of critical points is dominated by a single term, proportional to the second-order Wiener chaos projection $\mathcal{N}_{\ell ; I}^{c}[2]$, and 
b) the second-order Wiener chaos projection $\mathcal{N}_{\ell ; I}^{c}[2]$ admits a simple expression in terms of the integral of $H_{2}\left(f_{\ell}(x)\right)$ over $\mathbb{S}^{2}$.

These results share some surprising features with the asymptotic expressions for Lipschitz-Killing curvatures reported in (1.2 1.4); in particular, while the computation of critical points by means of the Kac-Rice formula (see below) requires the evaluation of gradient and Hessian fields, the dominating term depends in the highfrequency regime only on the (random) $L^{2}\left(\mathbb{S}^{2}\right)$ norm of the eigenfunctions $f_{\ell}$ :

$$
\frac{4 \pi}{2 \ell+1} \sum_{m=-\ell}^{\ell}\left\{\left|a_{\ell m}\right|^{2}-1\right\}=\int_{\mathbb{S}^{2}} H_{2}\left(f_{\ell}(x)\right) d x=\int_{\mathbb{S}^{2}} f_{\ell}^{2}(x) d x-4 \pi=\left\|f_{\ell}\right\|_{L^{2}\left(\mathbb{S}^{2}\right)}^{2}-\mathbb{E}\left\|f_{\ell}\right\|_{L^{2}\left(\mathbb{S}^{2}\right)}^{2} .
$$

As a simple Corollary we are able to establish a quantitative Central Limit Theorem; for this purpose, let us recall the definition of the Wasserstein distance between probability distributions (see for instance [30] and the references therein), which for any two random variables $X, Y$ is given by

$$
d_{W}(X, Y):=\sup _{h \in \operatorname{Lip}(1)}|\mathbb{E} h(X)-\mathbb{E} h(Y)|,
$$

where Lip(1) denotes the space of Lipschitz functions with bounding constant equal to 1 . Writing $Z \sim \mathcal{N}(0,1)$ for a standard Gaussian variable, we have that:

Corollary 2. For $I \subset \mathbb{R}$ s.t. $\nu^{c}(I) \neq 0$, as $\ell \rightarrow \infty$,

$$
d_{W}\left(\frac{\mathcal{N}_{\ell}^{c}(I)-\mathbb{E}\left[\mathcal{N}_{\ell}^{c}(I)\right]}{\sqrt{\operatorname{Var}\left(\mathcal{N}_{\ell}^{c}(I)\right)}}, Z\right)=o(1) .
$$

The proof of the Corollary is standard, noting that by (1.5) the right hand side of (1.10) is proportional to a sum of independent and identically distributed random variables with zero mean and finite variance, plus a remainder which is negligible in the mean square sense.

Remark 1.2. Note that for $I=[u, \infty)$ we have that

$$
\begin{aligned}
\int_{u}^{\infty} p_{3}^{c}(t) d t & =\int_{u}^{\infty} \frac{1}{\sqrt{8 \pi}} e^{-\frac{3}{2} t^{2}}\left(2-6 t^{2}\right) d t-\int_{u}^{\infty} \frac{1}{\sqrt{8 \pi}} e^{-\frac{1}{2} t^{2}}\left(1-4 t^{2}+t^{4}\right) d t \\
& =-\frac{1}{2} \frac{\sqrt{2}}{\sqrt{\pi}} u e^{-\frac{3}{2} u^{2}}+\frac{1}{4} \frac{\sqrt{2}}{\sqrt{\pi}} u e^{-\frac{1}{2} u^{2}}\left(u^{2}-1\right)
\end{aligned}
$$

and hence

$$
\mathcal{N}_{\ell}^{c}(I)-\mathbb{E}\left[\mathcal{N}_{\ell}^{c}(I)\right]=\frac{\lambda_{\ell}}{2}\left\{-\frac{\sqrt{2}}{\sqrt{3}} H_{1}\left(\frac{\sqrt{3}}{\sqrt{2}} u\right) \phi\left(\frac{\sqrt{3}}{\sqrt{2}} u\right)+\frac{1}{2} H_{1}(u) H_{2}(u) \phi(u)\right\} \frac{1}{2 \pi} \int_{\mathbb{S}^{2}} H_{2}\left(f_{\ell}(x)\right) d x+o_{p}\left(\ell^{3 / 2}\right) .
$$

1.3. Discussion. Theorem 1 yields one more reduction principle for geometric functionals of random spherical harmonics, entailing in particular the asymptotically full correlation

$$
\lim _{\ell \rightarrow \infty} \operatorname{Corr}\left\{\mathcal{N}_{\ell}^{c}(I), \mathcal{L}_{k}\left(A_{u}\left(f_{\ell} ; \mathbb{S}^{2}\right)\right)\right\}=1 \text { for all } u \neq 0, I \text { such that } \int_{I} p_{3}^{c}(t) d t \neq 0, \text { and } k=0,1,2 .
$$

It should be noted that $\int_{I} p_{3}^{c}(t) d t \neq 0$ holds for all half-intervals of the form $[u, \infty), u \neq 0, \pm \bar{u}$, where $\bar{u} \simeq 1.2096$ solves $-2 e^{-\bar{u}^{2}}+\left(\bar{u}^{2}-1\right)=0$. In other words, knowledge of the number of critical points on any excursion set $A_{u}$ for $u \neq 0, \pm \bar{u}$ allows to fully characterize (at least in the high-energy limit) the geometry of these excursion sets, i.e., their area, their boundary length, and their Euler-Poincaré characteristic. As mentioned earlier, it is also remarkable that, while the computation of the number of critical points requires the evaluation of higher-order derivatives, the asymptotic expression given in Theorem 1 depends on the $L^{2}$ norm of $f_{\ell}$ and no extra information.

An interesting open question is the characterization of the behaviour of critical points for intervals $I$ such that the integral of $p_{3}^{c}($.$) vanishes, the most interesting case being clearly I=\mathbb{R}$, i.e., the total number of critical points. A heuristic rationale explaining why the variance of the total number of critical points is asymptotically an order of magnitude smaller (up to logarithmic factors) than for "typical" intervals $I$ can be given as follows; from (1.11), Theorem 1 can be viewed as stating that the fluctuations in the number of critical points over a "generic" interval $I$ are proportional to the fluctuation in the random norm of the eigenfunctions. Clearly this cannot be the case for $I=\mathbb{R}$; indeed, the total number of critical points for a given realization $f_{\ell}$ is independent from any scaling factor, including the $L^{2}\left(\mathbb{S}^{2}\right)$ norm of the eigenfunctions. This leaves open the 
question about the asymptotic distribution for this total number $\mathcal{N}_{\ell}^{c}$; by analogy with some recent results by [25, we conjecture that the following expression holds:

$$
\mathcal{N}_{\ell}^{c}(I)-\mathbb{E}\left[\mathcal{N}_{\ell}^{c}(I)\right]=-\mu^{c}(I) \ell^{2} \frac{1}{4 !} \int_{\mathbb{S}^{2}} H_{4}\left(f_{\ell}(x)\right) d x+O_{p}\left(\ell^{2}\right) .
$$

Note that

$$
\begin{aligned}
\operatorname{Var}\left(-\mu^{c}(I) \ell^{2} \frac{1}{4 !} \int_{\mathbb{S}^{2}} H_{4}\left(f_{\ell}(x)\right) d x\right) & =\left[\mu^{c}(I)\right]^{2} \ell^{4} \frac{1}{576} \operatorname{Var}\left(\int_{\mathbb{S}^{2}} H_{4}\left(f_{\ell}(x)\right) d x\right) \\
& =\left[\mu^{c}(I)\right]^{2} \ell^{4} \frac{1}{576} \frac{576 \log \ell}{\ell^{2}}+O\left(\ell^{2}\right)=\left[\mu^{c}(I)\right]^{2} \ell^{2} \log \ell+O\left(\ell^{2}\right),
\end{aligned}
$$

consistent with (1.7). In particular, for the total number of critical points we conjecture the asymptotic equivalence

$$
\mathcal{N}_{\ell}^{c}-\mathbb{E}\left[\mathcal{N}_{\ell}^{c}\right]=-\frac{1}{3^{3 / 2} \pi} \ell^{2} \frac{1}{4 !} \int_{\mathbb{S}^{2}} H_{4}\left(f_{\ell}(x)\right) d x+O_{p}\left(\ell^{2}\right),
$$

the variance of the right-hand side being consistent with the result given in [9], where is shown that

$$
\operatorname{Var}\left(\mathcal{N}_{\ell}^{c}\right)=\frac{\ell^{2} \log \ell}{3^{3} \pi^{2}}+O\left(\ell^{2}\right)
$$

The investigation of this conjecture is left as a topic for further research.

1.4. Plan of the paper. As mentioned above, our proof requires two main ingredients, i.e., the Kac-Rice formula to express the number of critical points as a local functional of gradient and Hessian, and its expansion into Hermite polynomials/Wiener-Ito chaoses. For a correct implementation of the Kac-Rice formula, our first step is to review in Section 2 some background differential geometry material on the gradient and Hessian fields, and to compute their covariances; the properties of the resulting covariance matrices are then established in Section 3, where it is shown in particular that the covariance function for the gradient vector of random eigenfunctions evaluated at any two arbitrary points on the sphere is non-singular. These results are then used in Section 4 to prove the validity (in the $L^{2}(\Omega)$ sense) of the expansion for the Kac-Rice formula into Wiener chaoses, a technique exploited in other recent papers on geometric functionals of Gaussian eigenfunctions, for instance also in [27, 24], 23], 14, 32, 4], 10], 25]. Finally, in Section 5] the expansion is analytically computed and the simple dominating term is derived. A number of technical Lemmas related to computations of covariances and conditional expected values are collected in the Appendix.

\section{Gradient and Hessian Fields}

The proper computation of covariance matrices requires some careful discussion on (standard) background material in differential geometry. For $x=\left(\theta_{x}, \varphi_{x}\right) \in \mathbb{S}^{2} \backslash\{N, S\}(N, S$ are the north and south poles i.e. $\theta=0$ and $\theta=\pi$ respectively), the vectors

$$
\partial_{1 ; x}=\left.\frac{\partial}{\partial \theta}\right|_{\theta=\theta_{x}}, \quad \partial_{2 ; x}=\left.\frac{1}{\sin \theta} \frac{\partial}{\partial \varphi}\right|_{\theta=\theta_{x}, \varphi=\varphi_{x}},
$$

constitute an orthonormal basis for the tangent plane $T_{x}\left(\mathbb{S}^{2}\right)$; in these coordinates the gradient is given by $\nabla=\left(\frac{\partial}{\partial \theta}, \frac{1}{\sin \theta} \frac{\partial}{\partial \varphi}\right)$. For second-order derivatives, we shall use the following notation:

$$
\partial_{11}:=\frac{\partial^{2}}{\partial \theta^{2}}, \quad \partial_{21}:=\frac{\partial^{2}}{\sin \theta \partial \varphi \partial \theta}, \quad \partial_{22}:=\frac{\partial^{2}}{\sin ^{2} \theta \partial \varphi^{2}}
$$

Let us now recall that the covariant Hessian of a function $f \in C^{2}\left(\mathbb{S}^{2}\right)$ is the bilinear symmetric map from $C^{1}\left(T\left(\mathbb{S}^{2}\right)\right) \times C^{1}\left(T\left(\mathbb{S}^{2}\right)\right)$ to $C^{0}\left(\mathbb{S}^{2}\right)$ defined by

$$
\left(\nabla^{2} f\right)(X, Y)=X Y f-\nabla_{X} Y f, \quad X, Y \in T\left(\mathbb{S}^{2}\right)
$$

where $\nabla_{X}$ denotes Levi-Civita connection, see [1], Chapter 7 or the Appendix below for some details and definitions. For our computations to follow we shall need the matrix-valued process $\nabla^{2} f_{\ell}(x)$ with elements given by $\left\{\left(\nabla^{2} f\right)\left(\partial_{a}, \partial_{b}\right)\right\}_{a, b=1,2}$; in coordinates as above, this matrix can be expressed as (see Appendix A for some more details)

$$
\nabla^{2} f_{\ell}(x)=\left[\begin{array}{cc}
\frac{\partial^{2} f_{\ell}(x)}{\partial \theta^{2}} & \frac{1}{\sin \theta_{x}}\left[\frac{\partial^{2} f_{\ell}(x)}{\partial \theta \partial \varphi}-\frac{\cos \theta_{x}}{\sin \theta_{x}} \frac{\partial f_{\ell}(x)}{\partial \varphi}\right] \\
\frac{1}{\sin \theta_{x}}\left[\frac{\partial^{2} f_{\ell}(x)}{\partial \theta \partial \varphi}-\frac{\cos \theta_{x}}{\sin \theta_{x}} \frac{\partial f_{\ell}(x)}{\partial \varphi}\right] & \frac{1}{\sin ^{2} \theta_{x}}\left[\frac{\partial^{2} f_{\ell}(x)}{\partial \varphi^{2}}+\sin \theta_{x} \cos \theta \frac{\partial f_{\ell}(x)}{\partial \theta}\right]
\end{array}\right]
$$




$$
=\left[\begin{array}{cc}
\partial_{11} f_{\ell}(x) & \left(\partial_{21}-\cot \theta_{x} \partial_{2}\right) f_{\ell}(x) \\
\left(\partial_{21}-\cot \theta_{x} \partial_{2}\right) f_{\ell}(x) & \left(\partial_{22}+\cot \theta_{x} \partial_{1}\right) f_{\ell}(x)
\end{array}\right]
$$

We write as usual $\operatorname{vec}\left(\nabla^{2} f_{\ell}\right)$ for the column vector stacking the different elements of $\left(\nabla^{2} f_{\ell}\right)$, i.e.,

$$
\operatorname{vec}\left(\nabla^{2} f_{\ell}\right)=\left(\begin{array}{c}
\partial_{11} f_{\ell}(x) \\
\partial_{21} f_{\ell}(x)-\cot \theta_{x} \partial_{2} f_{\ell}(x) \\
\partial_{22} f_{\ell}(x)+\cot \theta_{x} \partial_{1} f_{\ell}(x)
\end{array}\right)
$$

The next result gives the exact covariance matrix for the five-dimensional vector including the elements of the gradient and the (covariant) Hessian:

Proposition 3. For every point $x \in \mathbb{S}^{2} \backslash\{N ; S\}$, the distribution of the 5 -dimensional vector $\left(\nabla f_{\ell}, v e c\left(\nabla^{2} f_{\ell}\right)\right)$ is zero-mean Gaussian, with variance-covariance matrix

$$
\left(\begin{array}{ccccc}
P_{\ell}^{\prime}(1) & 0 & 0 & 0 & 0 \\
0 & P_{\ell}^{\prime}(1) & 0 & 0 & 0 \\
0 & 0 & 3 P_{\ell}^{\prime \prime}(1)+P_{\ell}^{\prime}(1) & 0 & P_{\ell}^{\prime \prime}(1)+P_{\ell}^{\prime}(1) \\
0 & 0 & 0 & P_{\ell}^{\prime \prime}(1) & 0 \\
0 & 0 & P_{\ell}^{\prime \prime}(1)+P_{\ell}^{\prime}(1) & 0 & 3 P_{\ell}^{\prime \prime}(1)+P_{\ell}^{\prime}(1)
\end{array}\right)
$$

Proof. The following results are proved in Lemma 11 in Appendix B:

$$
\operatorname{Var}\left(\partial_{1} f_{\ell}(x)\right)=\operatorname{Var}\left(\partial_{2} f_{\ell}(x)\right)=P_{\ell}^{\prime}(1), \quad \operatorname{Cov}\left(\partial_{1} f_{\ell}(x), \partial_{1} f_{\ell}(x)\right)=0,
$$

for the higher order derivatives, we have (see Lemmas 15, 20, 18, 16, 19, 17)

$$
\begin{aligned}
\operatorname{Var}\left(\partial_{11} f_{\ell}(x)\right) & =3 P_{\ell}^{\prime \prime}(1)+P_{\ell}^{\prime}(1), \\
\operatorname{Var}\left(\partial_{21} f_{\ell}(x)\right) & =P_{\ell}^{\prime \prime}(1)+\cot ^{2} \theta_{x} P_{\ell}^{\prime}(1), \\
\operatorname{Var}\left(\partial_{22} f_{\ell}(x)\right) & =3 P_{\ell}^{\prime \prime}(1)+P_{\ell}^{\prime}(1)-\cot ^{2} \theta_{x} P_{\ell}^{\prime}(1),
\end{aligned}
$$

and

$$
\begin{aligned}
& \operatorname{Cov}\left(\partial_{22} f_{\ell}(x), \partial_{11} f_{\ell}(x)\right)=P_{\ell}^{\prime \prime}(1)+P_{\ell}^{\prime}(1), \\
& \operatorname{Cov}\left(\partial_{22} f_{\ell}(x), \partial_{21} f_{\ell}(x)\right)=\operatorname{Cov}\left(\partial_{11} f_{\ell}(x), \partial_{21} f_{\ell}(x)\right)=0 .
\end{aligned}
$$

Moreover in Lemmas 12, 14, 13, it is shown that

$$
\begin{aligned}
& \operatorname{Cov}\left(\partial_{11} f_{\ell}(x), \partial_{1} f_{\ell}(x)\right)=\operatorname{Cov}\left(\partial_{11} f_{\ell}(x), \partial_{2} f_{\ell}(x)\right)=0, \\
& \operatorname{Cov}\left(\partial_{21} f_{\ell}(x), \partial_{1} f_{\ell}(x)\right)=0, \operatorname{Cov}\left(\partial_{21} f_{\ell}(x), \partial_{2} f_{\ell}(x)\right)=\cot \theta_{x} P_{\ell}^{\prime}(1), \\
& \operatorname{Cov}\left(\partial_{22} f_{\ell}(x), \partial_{1} f_{\ell}(x)\right)=-\cot \theta_{x} P_{\ell}^{\prime}(1), \operatorname{Cov}\left(\partial_{22} f_{\ell}(x), \partial_{2} f_{\ell}(x)\right)=0 .
\end{aligned}
$$

The remaining computations are all straightforward - it is interesting, however, to note the delicate cancellations that occur. For instance, we have, for any $x \in \mathbb{S}^{2}$,

$$
\begin{aligned}
& \operatorname{Var}\left(\frac{1}{\sin ^{2} \theta_{x}} \frac{\partial^{2} f_{\ell}(x)}{\partial \varphi^{2}}+\cot \theta_{x} \frac{\partial f_{\ell}(x)}{\partial \theta}\right) \\
= & \operatorname{Var}\left(\partial_{22} f_{\ell}(x)\right)+\cot ^{2} \theta_{x} \operatorname{Var}\left(\partial_{1} f_{\ell}(x)\right)+2 \cot \theta_{x} \operatorname{Cov}\left(\partial_{22} f_{\ell}(x), \partial_{2} f_{\ell}(x)\right) \\
= & 3 P_{\ell}^{\prime \prime}(1)+P_{\ell}^{\prime}(1)+\cot ^{2} \theta_{x} P_{\ell}^{\prime}(1)+\cot ^{2} \theta_{x} P_{\ell}^{\prime}(1)-2 \cot ^{2} \theta_{x} P_{\ell}^{\prime}(1) \\
= & 3 P_{\ell}^{\prime \prime}(1)+P_{\ell}^{\prime}(1) .
\end{aligned}
$$

Likewise

$$
\begin{aligned}
& \operatorname{Var}\left(\frac{1}{\sin \theta} \frac{\partial^{2} f_{\ell}(x)}{\partial \theta \partial \varphi}-\cot \theta_{x} \frac{1}{\sin \theta_{x}} \frac{\partial f_{\ell}(x)}{\partial \varphi}\right) \\
& =\operatorname{Var}\left(\partial_{12} f_{\ell}(x)+\cot ^{2} \theta_{x} \operatorname{Var}\left(\partial_{2} f_{\ell}(x)\right)-2 \cot \theta_{x} \operatorname{Cov}\left(\partial_{21} f_{\ell}(x), \partial_{2} f_{\ell}(x)\right)\right. \\
& =P_{\ell}^{\prime \prime}(1)+\cot ^{2} \theta_{x} P_{\ell}^{\prime}(1)+\cot ^{2} \theta_{x} P_{\ell}^{\prime}(1)-2 \cot ^{2} \theta_{x} P_{\ell}^{\prime}(1) \\
& =P_{\ell}^{\prime \prime}(1) .
\end{aligned}
$$

All the remaining terms are immediate consequences of the Lemmas. 
Remark 2.1. It follows from the previous proposition that the elements of the covariant Hessian (the secondorder covariant derivatives) have finite and constant variance for all locations on the sphere. It should be stressed that this is not the case for the standard derivatives, i.e. the elements of the iterated gradient $\nabla^{2} f_{\ell}$; in fact, we have shown above that

$$
\begin{aligned}
\operatorname{Var}\left(\partial_{11} f_{\ell}(x)\right) & =3 P_{\ell}^{\prime \prime}(1)+P_{\ell}^{\prime}(1), \\
\operatorname{Var}\left(\partial_{21} f_{\ell}(x)\right) & =P_{\ell}^{\prime \prime}(1)+2 \cot ^{2} \theta_{x} P_{\ell}^{\prime}(1), \\
\operatorname{Var}\left(\partial_{22} f_{\ell}(x)\right) & =3 P_{\ell}^{\prime \prime}(1)+P_{\ell}^{\prime}(1)-\cot ^{2} \theta_{x} P_{\ell}^{\prime}(1),
\end{aligned}
$$

so that the variance of $\left(\partial_{12} f_{\ell}(x), \partial_{22} f_{\ell}(x)\right)$ is not constant over $\mathbb{S}^{2}$ - and indeed not even bounded, so that $L^{2}$ expansions would not be feasible. On the other hand, in the element of the (covariant) Hessian the extra terms introduced by means of the Levi-Civita connection and the Christoffel symbols ensure the exact cancellation of the location-dependent factors. Note also that while covariant derivatives of different orders are zero when evaluated on the same point, this is not the case for standard derivatives, indeed both

$$
\operatorname{Cov}\left(\partial_{21} f_{\ell}(x), \partial_{2} f_{\ell}(x)\right)=-\operatorname{Cov}\left(\partial_{22} f_{\ell}(x), \partial_{1} f_{\ell}(x)\right)=\cot \theta_{x} P_{\ell}^{\prime}(1) \text {. }
$$

These covariances are zero only for $\theta_{x}=\frac{\pi}{2}$, where the tangent plane has a "Euclidean" basis $\frac{\partial}{\partial \theta}$, $\frac{\partial}{\partial \varphi}$ and the covariant derivatives take the same values as the standard ones.

Remark 2.2. Note that each one of the three quantities

$$
\operatorname{Var}\left(\frac{\partial^{2} f_{\ell}}{\partial \theta^{2}}\right), \quad \operatorname{Var}\left(\frac{1}{\sin ^{2} \theta} \frac{\partial^{2} f_{\ell}}{\partial \varphi^{2}}+\cot \theta \frac{\partial f_{\ell}}{\partial \theta}\right), \quad \operatorname{Cov}\left(\frac{\partial^{2} f_{\ell}}{\partial \theta^{2}}, \frac{1}{\sin ^{2} \theta} \frac{\partial^{2} f_{\ell}}{\partial \varphi^{2}}+\cot \theta \frac{\partial f_{\ell}}{\partial \theta}\right),
$$

could actually be evaluated immediately from the other two. Indeed, recalling that the trace of the Hessian matrix is indeed the spherical Laplacian

$$
\Delta_{\mathbb{S}^{2}} f_{\ell}(x)=\frac{\partial^{2} f_{\ell}}{\partial \theta^{2}}+\frac{1}{\sin ^{2} \theta} \frac{\partial^{2} f_{\ell}}{\partial \varphi^{2}}+\cot \theta \frac{\partial f_{\ell}}{\partial \theta}=-\lambda_{\ell} f_{\ell}(x)
$$

we have the identity

$$
\begin{gathered}
\lambda_{\ell}^{2}=\operatorname{Var}\left(-\lambda_{\ell} f_{\ell}\right)=\operatorname{Var}\left(\frac{\partial^{2} f_{\ell}}{\partial \theta^{2}}+\frac{1}{\sin ^{2} \theta} \frac{\partial^{2} f_{\ell}}{\partial \varphi^{2}}+\cot \theta \frac{\partial f_{\ell}}{\partial \theta}\right) \\
=\operatorname{Var}\left(\frac{\partial^{2} f_{\ell}}{\partial \theta^{2}}\right)+\operatorname{Var}\left(\frac{1}{\sin ^{2} \theta} \frac{\partial^{2} f_{\ell}}{\partial \varphi^{2}}+\cot \theta \frac{\partial f_{\ell}}{\partial \theta}\right)+2 \operatorname{Cov}\left(\frac{\partial^{2} f_{\ell}}{\partial \theta^{2}}, \frac{1}{\sin ^{2} \theta} \frac{\partial^{2} f_{\ell}}{\partial \varphi^{2}}+\cot \theta \frac{\partial f_{\ell}}{\partial \theta}\right),
\end{gathered}
$$

whence, for instance, using

$$
\begin{gathered}
\operatorname{Var}\left(\frac{\partial^{2} f_{\ell}}{\partial \theta^{2}}\right)=3 P_{\ell}^{\prime \prime}(1)+P_{\ell}^{\prime}(1) \\
\operatorname{Cov}\left(\frac{\partial^{2} f_{\ell}}{\partial \theta^{2}}, \frac{1}{\sin ^{2} \theta} \frac{\partial^{2} f_{\ell}}{\partial \varphi^{2}}+\sin \theta \cos \theta \frac{\partial f_{\ell}}{\partial \theta}\right)=P_{\ell}^{\prime \prime}(1)+P_{\ell}^{\prime}(1),
\end{gathered}
$$

we obtain immediately

$$
\begin{gathered}
\operatorname{Var}\left(\frac{1}{\sin ^{2} \theta} \frac{\partial^{2} f_{\ell}}{\partial \varphi^{2}}+\cot \theta \frac{\partial f_{\ell}}{\partial \theta}\right) \\
=\lambda_{\ell}^{2}-3 P_{\ell}^{\prime \prime}(1)-P_{\ell}^{\prime}(1)-2 P_{\ell}^{\prime \prime}(1)-2 P_{\ell}^{\prime}(1)=\lambda_{\ell}^{2}-5 P_{\ell}^{\prime \prime}(1)-3 P_{\ell}^{\prime}(1) \\
=\lambda_{\ell}^{2}-5 \frac{\lambda_{\ell}}{8}\left(\lambda_{\ell}-2\right)-\frac{3}{2} \lambda_{\ell}=3 \frac{\lambda_{\ell}}{8}\left(\lambda_{\ell}-2\right)+\frac{\lambda_{\ell}}{2} \\
=3 P_{\ell}^{\prime \prime}(1)+P_{\ell}^{\prime}(1) .
\end{gathered}
$$

\section{Covariance Matrices}

Recall first that, since the $f_{\ell}$ are eigenfunctions of the spherical Laplacian, hence, for every $x \in \mathbb{S}^{2}$, we can write

$$
f_{\ell}(x)=-\frac{\Delta_{\mathbb{S}^{2}} f_{\ell}(x)}{\lambda_{\ell}}
$$

note that at the critical points we have $\Delta_{\mathbb{S}^{2}} f_{\ell}=\partial_{11} f_{\ell}+\partial_{22} f_{\ell}$, whence their number with value in $I$ is, by symmetry, given by

$$
\mathcal{N}_{\ell}^{c}(I)=\#\left\{x \in \mathbb{S}^{2}: \frac{\Delta_{\mathbb{S}^{2}} f_{\ell}(x)}{\lambda_{\ell}} \in I, \quad \nabla f_{\ell}(x)=0\right\}=\#\left\{x \in \mathbb{S}^{2}: \frac{\partial_{11} f_{\ell}+\partial_{22} f_{\ell}}{\lambda_{\ell}} \in I, \quad \nabla f_{\ell}(x)=0\right\} .
$$


For every $x \in \mathbb{S}^{2}$, let us now denote by $\Sigma_{\ell}(x, y)$ the covariance matrix for the 10-dimensional Gaussian random vector

$$
\left(\nabla f_{\ell}(x), \nabla f_{\ell}(y), \operatorname{vec}\left(\nabla^{2} f_{\ell}(x)\right), \operatorname{vec}\left(\nabla^{2} f_{\ell}(y)\right)\right),
$$

which combines the gradient and the elements of the Hessian evaluated at $x, y$; we shall write

$$
\Sigma_{\ell}(x, y)=\left(\begin{array}{cc}
A_{\ell}(x, y) & B_{\ell}(x, y) \\
B_{\ell}^{T}(x, y) & C_{\ell}(x, y)
\end{array}\right)
$$

where the $A_{\ell}$ and $C_{\ell}$ components collect the variances of the gradient and Hessian terms respectively, while the matrix $B_{\ell}$ collects the covariances between first and second order covariant derivatives. More explicitly, we have that

$$
A_{\ell}(x, y)_{4 \times 4}=\left(\begin{array}{ll}
a_{\ell}(x, x) & a_{\ell}(x, y) \\
a_{\ell}(y, x) & a_{\ell}(y, y)
\end{array}\right)=\left.\mathbb{E}\left[\left(\begin{array}{c}
\nabla f_{\ell}(\bar{x})^{T} \\
\nabla f_{\ell}(\bar{y})^{T}
\end{array}\right)\left(\begin{array}{ll}
\nabla f_{\ell}(x) & \nabla f_{\ell}(y)
\end{array}\right)\right]\right|_{x=\bar{x}, y=\bar{y}},
$$

and more precisely

$$
a_{\ell}(x, x)=a_{\ell}(y, y)=\left(\begin{array}{cc}
P_{\ell}^{\prime}(1) & 0 \\
0 & P_{\ell}^{\prime}(1)
\end{array}\right) .
$$

Recalling also that $P_{\ell}^{\prime}(1)=\frac{\ell(\ell+1)}{2}$, for $\lambda_{\ell}=\ell(\ell+1)$, we have

$$
A_{\ell}(x, y)=\left(\begin{array}{cccc}
\frac{\lambda_{\ell}}{2} & 0 & \alpha_{1, \ell}(x, y) & 0 \\
0 & \frac{\lambda_{\ell}}{2} & 0 & \alpha_{2, \ell}(x, y) \\
\alpha_{1, \ell}(x, y) & 0 & \frac{\lambda_{\ell}}{2} & 0 \\
0 & \alpha_{2, \ell}(x, y) & 0 & \frac{\lambda_{\ell}}{2}
\end{array}\right) .
$$

The matrix $B_{\ell}$ collects the covariances between first and second order derivatives, and is given by

$$
\begin{aligned}
& B_{\ell}(x, y)_{4 \times 6}=\left.\mathbb{E}\left[\left(\begin{array}{c}
\operatorname{vec}\left(\nabla f_{\ell}(\bar{x})\right) \\
\operatorname{vec}\left(\nabla f_{\ell}(\bar{y})\right)
\end{array}\right)\left(\operatorname{vec}\left(\nabla^{2} f_{\ell}(x)\right)^{T} \quad \operatorname{vec}\left(\nabla^{2} f_{\ell}(y)\right)^{T}\right)\right]\right|_{x=\bar{x}, y=\bar{y}} \\
& =\left(\begin{array}{ll}
b_{\ell}(x, x) & b_{\ell}(x, y) \\
b_{\ell}(y, x) & b_{\ell}(y, y)
\end{array}\right) .
\end{aligned}
$$

It is well-known that for Gaussian isotropic processes, for $i, j=1,2$, the second derivatives $e_{i}^{x} e_{j}^{x} f_{\ell}(x)$ are independent of $e_{i}^{x} f_{\ell}(x)$ at every fixed point $x \in \mathbb{S}^{2}$ see, e.g., [1] Section 5.5; we have then

$$
b_{\ell}(x, x)=b_{\ell}(y, y)=\left(\begin{array}{lll}
0 & 0 & 0 \\
0 & 0 & 0
\end{array}\right) .
$$

Finally, the matrix $C_{\ell}$ contains the variances of second-order derivatives, and we have

$$
C_{\ell}(x, y)_{6 \times 6}=\left.\mathbb{E}\left[\left(\begin{array}{c}
\nabla^{2} f_{\ell}(\bar{x})^{T} \\
\nabla^{2} f_{\ell}(\bar{y})^{T}
\end{array}\right)\left(\begin{array}{ll}
\nabla^{2} f_{\ell}(x) & \nabla^{2} f_{\ell}(\bar{y})
\end{array}\right)\right]\right|_{x=\bar{x}, y=\bar{y}}=\left(\begin{array}{ll}
c_{\ell}(x, x) & c_{\ell}(x, y) \\
c_{\ell}(y, x) & c_{\ell}(y, y)
\end{array}\right) .
$$

From direct calculations and the formula $P_{\ell}^{\prime \prime}(1)=\frac{\lambda_{\ell}}{8}\left(\lambda_{\ell}-2\right)$, it immediately follows that

$$
\begin{aligned}
c_{\ell}(x, x) & =\left(\begin{array}{ccc}
3 P_{\ell}^{\prime \prime}(1)+P_{\ell}^{\prime}(1) & 0 & P_{\ell}^{\prime \prime}(1)+P_{\ell}^{\prime}(1) \\
0 & P_{\ell}^{\prime \prime}(1) & 0 \\
P_{\ell}^{\prime \prime}(1)+P_{\ell}^{\prime}(1) & 0 & 3 P_{\ell}^{\prime \prime}(1)+P_{\ell}^{\prime}(1)
\end{array}\right) \\
& =\left(\begin{array}{ccc}
\frac{\lambda_{\ell}}{8}\left[3 \lambda_{\ell}-2\right] & 0 & \frac{\lambda_{\ell}}{8}\left[\lambda_{\ell}+2\right] \\
0 & \frac{\lambda_{\ell}}{8}\left[\lambda_{\ell}-2\right] & 0 \\
\frac{\lambda_{\ell}}{8}\left[\lambda_{\ell}+2\right] & 0 & \frac{\lambda_{\ell}}{8}\left[3 \lambda_{\ell}-2\right]
\end{array}\right)=c_{\ell}(y, y) .
\end{aligned}
$$

The following Proposition is really a special case of Lemma C1 in [36]; nevertheless we include a short proof for completeness:

Proposition 4. (See [36]) For every $(x, y) \in \mathbb{S}^{2}$ such that $d_{\mathbb{S}^{2}}(x, y) \neq 0, \pi$, the Gaussian vector $\left(\nabla f_{\ell}(x), \nabla f_{\ell}(y)\right)$ has a non-degenerate density function, i.e., the covariance matrix $A_{\ell}(x, y)$ is invertible. 
Proof. We compute the covariance matrix at the equator, where our choice of coordinates is such that both the vectors $\partial_{1}$ and $\partial_{2}$ are moved by parallel transport from $x$ to $y$. Given however any arbitrary orientations in $x$ and $y$, we would obtain the matrix $R(\psi) A_{\ell}(x, y) R(\psi)^{\prime}$, where

$$
R(\psi)=\left(\begin{array}{cccc}
1 & 0 & 0 & 0 \\
0 & 1 & 0 & 0 \\
0 & 0 & \cos \psi & -\sin \psi \\
0 & 0 & \sin \psi & \cos \psi
\end{array}\right)
$$

is the matrix that describes the rotation between $\partial_{a, x}$ and $\partial_{b, y}$ after transportation, $a, b=1,2$ (i.e., $\psi$ is the angle that occurs between $\partial_{1, x}$ when it is transported to $y$ along a geodesic in such a way that it remains parallel and the vector $\partial_{1, y}$; this angle is the same for both vectors because the basis are orthonormal). Clearly the matrices $R$ are full rank and hence have no impact on the rank.

We are now in the position to describe explicitly the symmetric matrix $A_{\ell}(x, y)$; for notational simplicity and without loss of generality, we focus on pairs of points lying on the "equator" $\theta_{x}=\theta_{y}=\frac{\pi}{2}$, where we obtain

$$
A_{\ell}(x, y)=\left(\begin{array}{cccc}
P_{\ell}^{\prime}(1) & * & * & * \\
0 & P_{\ell}^{\prime}(1) & * & * \\
P_{\ell}^{\prime}(\langle x, y\rangle) & 0 & P_{\ell}^{\prime}(1) & * \\
0 & -P_{\ell}^{\prime \prime}(\langle x, y\rangle) \sin ^{2}\left(\varphi_{x}-\varphi_{y}\right)+P_{\ell}^{\prime}(\langle x, y\rangle) \cos \left(\varphi_{x}-\varphi_{y}\right) & 0 & P_{\ell}^{\prime}(1)
\end{array}\right)
$$

with determinant equal to

$$
\begin{aligned}
& \left\{P_{\ell}^{\prime}(1)\right\}^{2}\left[\left\{P_{\ell}^{\prime}(1)\right\}^{2}-\left\{P_{\ell}^{\prime \prime}(\langle x, y\rangle) \sin ^{2}\left(\varphi_{x}-\varphi_{y}\right)+P_{\ell}^{\prime}(\langle x, y\rangle) \cos \left(\varphi_{x}-\varphi_{y}\right)\right\}^{2}\right] \\
& -\left\{P_{\ell}^{\prime}(\langle x, y\rangle)\right\}^{2}\left[\left\{P_{\ell}^{\prime}(1)\right\}^{2}-\left\{P_{\ell}^{\prime \prime}(\langle x, y\rangle) \sin ^{2}\left(\varphi_{x}-\varphi_{y}\right)+P_{\ell}^{\prime}(\langle x, y\rangle) \cos \left(\varphi_{x}-\varphi_{y}\right)\right\}^{2}\right] \\
& =\left[\left\{P_{\ell}^{\prime}(1)\right\}^{2}-\left[P_{\ell}^{\prime}(\langle x, y\rangle)\right\}^{2}\right]\left\{\left\{P_{\ell}^{\prime}(1)\right\}^{2}-\left\{P_{\ell}^{\prime \prime}(\langle x, y\rangle) \sin ^{2}\left(\varphi_{x}-\varphi_{y}\right)+P_{\ell}^{\prime}(\langle x, y\rangle) \cos \left(\varphi_{x}-\varphi_{y}\right)\right\}^{2}\right] .
\end{aligned}
$$

Now

$$
\left\{P_{\ell}^{\prime}(1)\right\}^{2}-\left\{P_{\ell}^{\prime}(\langle x, y\rangle)\right\}^{2}>0 \text { for all } x \neq y
$$

by standard properties of Legendre polynomials derivatives, which have a unique maximum at $u=1$. To prove the latter statement, assume by contradiction that $P_{\ell}^{\prime}(1)=P_{\ell}^{\prime}(\langle x, y\rangle)$ for some $x \neq y$; then we should have some $\alpha$ of modulus unity and some $\varphi$ such that

$$
\partial_{1, x} f_{\ell}=\sum_{m} a_{\ell m} P_{\ell m}^{\prime}(\cos \theta)=\alpha \sum_{m} a_{\ell m} P_{\ell m}^{\prime}(\cos \theta) \exp (i m \varphi)=\alpha \partial_{1, y} f_{\ell},
$$

the equality holding in $L^{2}(\omega)$, and hence with probability one; this conclusion is clearly impossible (for $\left.\varphi \neq 0, \pi\right)$, as it requires $\exp (i m \varphi)=1$ for all $m=-\ell, \ldots, \ell$. Likewise,

$$
\begin{aligned}
& \left\{P_{\ell}^{\prime}(1)\right\}^{2}-\left\{P_{\ell}^{\prime \prime}(\langle x, y\rangle) \sin ^{2}\left(\varphi_{x}-\varphi_{y}\right)+P_{\ell}^{\prime}(\langle x, y\rangle) \cos \left(\varphi_{x}-\varphi_{y}\right)\right\}^{2} \\
& =\operatorname{Var}\left(\partial_{1, x} f_{\ell}\right) \operatorname{Var}\left(\partial_{2, y} f_{\ell}\right)-\left\{\operatorname{Cov}\left(\partial_{2, x} f_{\ell}, \partial_{2, y} f_{\ell}\right)\right\}^{2}>0
\end{aligned}
$$

unless

$$
\partial_{2, y} f_{\ell}=\alpha \partial_{2, x} f_{\ell}, \text { for some } \alpha \text { such that }|\alpha|=1,
$$

for some $x, y$. However we know that, for $\theta_{x}=\theta_{y}=\frac{\pi}{2}$,

$$
\begin{aligned}
\partial_{2, y} f_{\ell} & =\sum_{m=-\ell}^{\ell} i m a_{\ell m} Y_{\ell m}\left(\theta_{y}, \varphi_{y}\right) \\
& =\sum_{m=-\ell}^{\ell} i m a_{\ell m} e^{i m \varphi_{y}} \sqrt{\frac{2 \ell+1}{4 \pi}} \sqrt{\frac{(\ell-m) !}{(\ell+m) !}} P_{\ell m}(0),
\end{aligned}
$$

and

$$
\partial_{2, x} f_{\ell}=\sum_{m=-\ell}^{\ell} i m a_{\ell m} e^{i m \varphi_{x}} \sqrt{\frac{2 \ell+1}{4 \pi}} \sqrt{\frac{(\ell-m) !}{(\ell+m) !}} P_{\ell m}(0),
$$

whence the identity (3.2) requires, for all $m$ such that $\ell+m$ is even (which ensures that $P_{\ell m}(0) \neq 0$ ),

$$
e^{i m \varphi_{y}}=\alpha e^{i m \varphi_{x}},
$$

as before impossible, unless $\varphi_{x}=\varphi_{y}$. Hence the statement of the Proposition follows. 


\section{KaC-Rice Formula and $L^{2}$-Convergence}

We can now build an approximating sequence of functions $\left(\mathcal{N}_{\ell, \varepsilon}^{c}(I)\right.$, say), and establish their convergence both in the $\omega$-almost sure and in the $L^{2}(\Omega)$ sense to $\mathcal{N}_{\ell}^{c}(I)$. More precisely, let $\delta_{\varepsilon}: \mathbb{R}^{2} \rightarrow \mathbb{R}$ be such that

$$
\delta_{\varepsilon}(z):=\frac{1}{\varepsilon^{2}} \mathbb{I}_{\{z \in[-\varepsilon / 2, \varepsilon / 2]\}},
$$

and define the approximating sequence

$$
\mathcal{N}_{\ell, \varepsilon}^{c}(I):=\int_{\mathbb{S}^{2}}\left|\operatorname{det}\left(\nabla^{2} f_{\ell}(x)\right)\right| \mathbb{I}_{\left\{f_{\ell}(x) \in I\right\}} \delta_{\varepsilon}\left(\nabla f_{\ell}(x)\right) d x ;
$$

it is possible to prove the almost sure and $L^{2}(\Omega)$ convergence of $\mathcal{N}_{\ell, \varepsilon}^{c}(I)$ to $\mathcal{N}_{\ell}^{c}(I)$ as $\varepsilon \rightarrow 0$ :

Lemma 5. For every $\ell \in \mathbb{N}$, we have

$$
\mathcal{N}_{\ell}^{c}(I)=\lim _{\varepsilon \rightarrow 0} \mathcal{N}_{\ell, \varepsilon}^{c}(I),
$$

where the convergence holds both $\omega$-a.s. and in $L^{2}(\Omega)$.

Proof. Step 1: Almost sure Convergence. To establish the convergence $\omega$-a.s., it is sufficient to refer to Theorem 11.2.3 in [1. Note that this Theorem refers to Euclidean domains, but the extension to the spherical case can be obtained by simply referring to local maps that form an atlas on the sphere, along the lines given for general manifolds again in [1, Theorem 12.1.1. Note also that these results are given for deterministic vector functions which are assumed to be continuous and nondegenerate (Morse), i.e. such that the Hessian does not vanish when the gradient does; these conditions are satisfied $\omega$-a.s. for Gaussian spherical harmonics.

Step 2: The Exact Kac-Rice Formula holds. The fact that $\mathbb{E}\left[\mathcal{N}_{\ell}^{c}(I)^{2}\right]<\infty$ has been shown in [7, exploiting an approximate Kac-Rice formula. It is possible here to give a stronger results; in particular, using Theorem 6.3 in [3] and Proposition 4, where we have shown that the determinant of the $4 \times 4$ covariance matrix $A_{\ell}(x, y)$ of first-order derivatives is strictly positive for every pair $(x, y) \in \mathbb{S}^{2} \times \mathbb{S}^{2}$, we have also that the exact Kac-Rice formula holds and hence

$$
\begin{gathered}
\mathbb{E}\left[\mathcal{N}_{\ell}^{c}(I)\left(\mathcal{N}_{\ell}^{c}(I)-1\right)\right] \\
=\frac{1}{(2 \pi)^{2}} \int_{\mathbb{S}^{2} \times \mathbb{S}^{2}} \mathbb{E}\left[\left|\operatorname{det}\left(\nabla^{2} f_{\ell}(x)\right)\right| \mid \operatorname{det}\left(\nabla^{2} f_{\ell}(y)\right) \| \nabla f_{\ell}(x)=\nabla f_{\ell}(y)=0\right] \mathbb{I}_{\left\{f_{\ell}(x) \in I\right\}} \mathbb{I}_{\left\{f_{\ell}(y) \in I\right\}} \frac{d x d y}{\sqrt{\operatorname{det}\left(A_{\ell}(x, y)\right)}} .
\end{gathered}
$$

Step 3: The $L^{2}$ Convergence of the Approximating Sequence. Because we have the almost sure convergence $\mathcal{N}_{\ell}^{c}(I)=\lim _{\varepsilon \rightarrow 0} \mathcal{N}_{\ell, \varepsilon}^{c}(I)$, to prove that $\mathbb{E}\left[\mathcal{N}_{\ell, \varepsilon}^{c}(I)-\mathcal{N}_{\ell}^{c}(I)\right]^{2} \rightarrow 0$ as $\varepsilon \rightarrow 0$ it is enough to show that

$$
\mathbb{E}\left[\left\{\mathcal{N}_{\ell, \varepsilon}^{c}(I)\right\}^{2}\right] \rightarrow \mathbb{E}\left[\left\{\mathcal{N}_{\ell}^{c}(I)\right\}^{2}\right] \text { as } \varepsilon \rightarrow 0 .
$$

Indeed we have

$$
\mathbb{E}\left[\left\{\mathcal{N}_{\ell, \varepsilon}^{c}(I)-\mathcal{N}_{\ell}^{c}(I)\right\}^{2}\right]=\mathbb{E}\left[\left\{\mathcal{N}_{\ell, \varepsilon}^{c}(I)\right\}^{2}\right]+\mathbb{E}\left[\left\{\mathcal{N}_{\ell}^{c}(I)\right\}^{2}\right]-2 \mathbb{E}\left[\mathcal{N}_{\ell, \varepsilon}^{c}(I) \mathcal{N}_{\ell}^{c}(I)\right],
$$

and $\mathbb{E}\left[\mathcal{N}_{\ell, \varepsilon}^{c}(I) \mathcal{N}_{\ell}^{c}(I)\right] \rightarrow \mathbb{E}\left[\left\{\mathcal{N}_{\ell}^{c}(I)\right\}^{2}\right]$ by Cauchy-Schwartz and Dominated Convergence. Recall first that by Federer's coarea formula

$$
\begin{aligned}
\mathcal{N}_{\ell, \varepsilon}^{c}(I) & =\int_{\mathbb{S}^{2}}\left|\operatorname{det}\left(\nabla^{2} f_{\ell}(x)\right)\right| \delta_{\varepsilon}\left(\nabla f_{\ell}(x)\right) \mathbb{I}_{\left\{f_{\ell}(x) \in I\right\}} d x \\
& =\int_{\mathbb{R}^{2}} \mathcal{N}_{\ell}\left(u_{1}, u_{2} ; I\right) \delta_{\varepsilon}\left(u_{1}, u_{2}\right) d u_{1} d u_{2}
\end{aligned}
$$

where

$$
\mathcal{N}_{\ell}\left(u_{1}, u_{2} ; I\right)=\operatorname{card}\left\{x \in \mathbb{S}^{2}: \nabla f_{\ell}(x)=\left(u_{1}, u_{2}\right), f_{\ell}(x) \in I\right\} .
$$

Now

$$
\begin{gathered}
\mathbb{E}\left[\left\{\mathcal{N}_{\ell}^{c}(I)\right\}^{2}\right] \leq \lim _{\varepsilon \rightarrow 0} \mathbb{E}\left[\left\{\mathcal{N}_{\ell, \varepsilon}^{c}(I)\right\}^{2}\right] \text { (by Fatou's Lemma) } \\
\left.=\liminf _{\varepsilon \rightarrow 0} \mathbb{E}\left[\left\{\int_{\mathbb{S}^{2}}\left|\operatorname{det}\left(\nabla^{2} f_{\ell}(x)\right)\right| \delta_{\varepsilon}\left(\nabla f_{\ell}(x)\right) \mathbb{I}_{\left\{f_{\ell}(x) \in I\right\}} d x\right\}^{2}\right] \text { (by definition of } \mathcal{N}_{\ell, \varepsilon}^{c}(I)\right) \\
=\liminf _{\varepsilon \rightarrow 0} \mathbb{E}\left[\left\{\int_{\mathbb{R}^{2}} \mathcal{N}_{\ell}\left(u_{1}, u_{2} ; I\right) \delta_{\varepsilon}\left(u_{1}, u_{2}\right) d u_{1} d u_{2}\right\}^{2}\right] \text { (by Federer's coarea formula) }
\end{gathered}
$$




$$
\begin{aligned}
& \leq \limsup _{\varepsilon \rightarrow 0} \mathbb{E}\left[\left\{\int_{\mathbb{R}^{2}} \mathcal{N}_{\ell}\left(u_{1}, u_{2} ; I\right) \delta_{\varepsilon}\left(u_{1}, u_{2}\right) d u_{1} d u_{2}\right\}^{2}\right] \text { (obvious) } \\
& \leq \limsup _{\varepsilon \rightarrow 0} \int_{\mathbb{R}^{2}} \mathbb{E}\left[\left\{\mathcal{N}_{\ell}\left(u_{1}, u_{2} ; I\right)\right\}^{2}\right] \delta_{\varepsilon}\left(u_{1}, u_{2}\right) d u_{1} d u_{2} \text { (by Jensen's inequality). }
\end{aligned}
$$

Clearly, if we will show that the application $\mathcal{A}: \mathbb{R}^{2} \rightarrow \mathbb{R}$ defined by $\mathcal{A}\left(u_{1}, u_{2}\right):=\mathbb{E}\left[\left\{\mathcal{N}_{\ell}\left(u_{1}, u_{2} ; I\right)\right\}^{2}\right]$ is continuous in a neighbourhood of the origin, we will be able to conclude that

$$
\limsup _{\varepsilon \rightarrow 0} \int_{\mathbb{R}^{2}} \mathbb{E}\left[\left\{\mathcal{N}_{\ell}\left(u_{1}, u_{2} ; I\right)\right\}^{2}\right] \delta_{\varepsilon}\left(u_{1}, u_{2}\right) d u_{1} d u_{2}=\mathbb{E}\left[\left\{\mathcal{N}_{\ell}^{c}(I)\right\}^{2}\right]
$$

and hence the result. Note however that

$$
\mathbb{E}\left[\left\{\mathcal{N}_{\ell}\left(u_{1}, u_{2} ; I\right)\right\}^{2}\right]=\int_{\mathbb{S}^{2} \times \mathbb{S}^{2}} K_{\ell}\left(x, y ; u_{1}, u_{2}\right) d x d y
$$

where we have introduced the (generalized) two-point correlation function

$$
K_{\ell}\left(x, y ; u_{1}, u_{2}\right):=\mathbb{E}\left[\left|\operatorname{det}\left(\nabla^{2} f_{\ell}(x)\right)\right| \mid \operatorname{det}\left(\nabla^{2} f_{\ell}(y)\right) \| \nabla f_{\ell}(x)=\nabla f_{\ell}(y)=\left(u_{1}, u_{2}\right)\right] p_{\left(\nabla f_{\ell}(x), \nabla f_{\ell}(y)\right)}\left(u_{1}, u_{2}\right) .
$$

Because $K_{\ell}\left(x, y ; u_{1}, u_{2}\right)$ is a linear combination of expectations of Gaussian moments, it is clearly continuous around $\left(u_{1}, u_{2}\right)=(0,0)$, where both its mean and variance are limited for every $(x \neq y)$. Hence if we can prove that $K_{\ell}\left(x, y ; u_{1}, u_{2}\right)$ is bounded, by the Lebesgue Dominated Convergence Theorem we shall have that

$$
\begin{aligned}
& \lim _{u_{1}, u_{2} \rightarrow 0} \mathbb{E}\left[\left\{\mathcal{N}_{\ell}\left(u_{1}, u_{2} ; I\right)\right\}^{2}\right]-\mathbb{E}\left[\left\{\mathcal{N}_{\ell}(0,0 ; I)\right\}^{2}\right] \\
& =\lim _{u_{1}, u_{2} \rightarrow 0} \int_{\mathbb{S}^{2} \times \mathbb{S}^{2}}\left\{K_{\ell}\left(x, y ; u_{1}, u_{2}\right)-K_{\ell}(x, y ; 0,0)\right\} d x d y=0,
\end{aligned}
$$

whence continuity follows. To prove that $K_{\ell}\left(x, y ; u_{1}, u_{2}\right)$ is bounded, we just need to generalize slightly Lemmas 4.5 and 4.6 from [7]; in particular, we have that

$$
p_{\left(\nabla f_{\ell}(x), \nabla f_{\ell}(y)\right)}\left(u_{1}, u_{2}\right) \leq\left\{\operatorname{det}\left(A_{\ell}(x, y)\right)\right\}^{-1 / 2} \leq \frac{C}{d_{\mathbb{S}^{2}}^{2}(x, y)}, \text { for some } C>0 \text {, for all }(x, y) \in \mathbb{S}^{2} \times \mathbb{S}^{2}
$$

in fact, this result was shown to hold in Lemma 4.5 of [7], but only for $d_{\mathbb{S}^{2}}(x, y)<c$, some $c>0$; however for $d_{\mathbb{S}^{2}}(x, y) \geq c$ we can use Proposition 4 to conclude that the determinant admits a non-zero minimum, because it is a strictly positive polynomial function on a compact set. Likewise, to bound

$$
\mathbb{E}\left[\left|\operatorname{det}\left(\nabla^{2} f_{\ell}(x)\right)\right| \mid \operatorname{det}\left(\nabla^{2} f_{\ell}(y)\right) \| \nabla f_{\ell}(x)=\nabla f_{\ell}(y)=\left(u_{1}, u_{2}\right)\right]
$$

we can argue exactly as in the proof of Lemma 4.6 in [7], the only difference being that the elements that appear in the Hessian matrices $\left\{\nabla^{2} f_{\ell}(x)\right\},\left\{\nabla^{2} f_{\ell}(y)\right\}$ have non-zero means; more precisely, let us write $\left(X_{1}, X_{2}, X_{3}, Y_{1}, Y_{2}, Y_{3}\right)$ for the six-dimensional vectors of second-order derivatives conditioned on $\nabla f_{\ell}(x)=$ $\nabla f_{\ell}(y)=\left(u_{1}, u_{2}\right)$ : it is a Gaussian vector with covariance matrix and expected value given respectively by

$$
\begin{aligned}
\Delta_{\ell}(x, y) & :=C_{\ell}(x, y)-B_{\ell}^{T}(x, y) A_{\ell}^{-1}(x, y) B_{\ell}(x, y) \\
\mu_{\ell}(x, y) & =\left(\begin{array}{c}
\mu_{1 \ell}(x, y) \\
\ldots \\
\mu_{6 \ell}(x, y)
\end{array}\right):=B_{\ell}^{T}(x, y) A_{\ell}^{-1}(x, y)\left(\begin{array}{l}
u_{1} \\
u_{2} \\
u_{1} \\
u_{2}
\end{array}\right) .
\end{aligned}
$$

Introduce also the centred six-dimensional vector

$$
\left(\widetilde{U}_{1}, \widetilde{U}_{2}, \widetilde{U}_{3}, \widetilde{U}_{4}, \widetilde{U}_{5}, \widetilde{U}_{6}\right)^{T}=\left(U_{1}, U_{2}, U_{3}, U_{4}, U_{5}, U_{6}\right)-\mu_{\ell}(x, y)
$$

Note that the elements of the vector $\left(\widetilde{U}_{1}, \widetilde{U}_{2}, \widetilde{U}_{3}, \widetilde{U}_{4}, \widetilde{U}_{5}, \widetilde{U}_{6}\right)$ can be interpreted as the second-order derivatives conditioned on the gradient being equal to zero. We show in Lemma 21 in Appendix $\mathrm{C}$ that for any fixed $\ell$

$$
\mu_{k \ell}(x, y)=O\left(d_{\mathbb{S}^{2}}(x, y)\right), k=1, \ldots, 6,
$$

and it is then readily verified that

$$
\begin{aligned}
& \mathbb{E}\left[\left|\operatorname{det}\left(\nabla^{2} f_{\ell}(x)\right)\right|\left|\operatorname{det}\left(\nabla^{2} f_{\ell}(y)\right)\right| \mid \nabla f_{\ell}(x)=\nabla f_{\ell}(y)=\left(u_{1}, u_{2}\right)\right] \\
= & \mathbb{E}\left[\left|\operatorname{det}\left(\begin{array}{cc}
\widetilde{U}_{1}+\mu_{1 \ell}(x, y) & \widetilde{U}_{2}+\mu_{2 \ell}(x, y) \\
\widetilde{U}_{2}+\mu_{2 \ell}(x, y) & \widetilde{U}_{3}+\mu_{3 \ell}(x, y)
\end{array}\right)\right|\left|\operatorname{det}\left(\begin{array}{cc}
\widetilde{U}_{4}+\mu_{4 \ell}(x, y) & \widetilde{U}_{5}+\mu_{5 \ell}(x, y) \\
\widetilde{U}_{5}+\mu_{5 \ell}(x, y) & \widetilde{U}_{6}+\mu_{6 \ell}(x, y)
\end{array}\right)\right|\right]
\end{aligned}
$$




$$
\begin{aligned}
& =\mathbb{E}\left[\left|\widetilde{U}_{1} \widetilde{U}_{3} \widetilde{U}_{4} \widetilde{U}_{6}\right|+\left|\widetilde{U}_{1} \widetilde{U}_{3} \widetilde{U}_{5}^{2}\right|+\left|\widetilde{U}_{2}^{2} \widetilde{U}_{4} \widetilde{U}_{6}\right|+\left|\widetilde{U}_{2}^{2} \widetilde{U}_{5}^{2}\right|\right]+O\left(d_{\mathbb{S}^{2}}^{2}(x, y)\right) \\
& =O\left(d_{\mathbb{S}^{2}}^{2}(x, y)\right)
\end{aligned}
$$

where for the last two steps we have used Lemma 4.5 in 7, that covers the behaviour of the moments for second order derivatives conditioned on $\nabla f_{\ell}(x)=\nabla f_{\ell}(y)=(0,0)$. Hence for all $(x, y) \in \mathbb{S}^{2} \times \mathbb{S}^{2}$, there exists some $C>0$, such that

$$
\mathbb{E}\left[\left|\operatorname{det}\left(\nabla^{2} f_{\ell}(x)\right)\right|\left|\operatorname{det}\left(\nabla^{2} f_{\ell}(y)\right)\right| \mid \nabla f_{\ell}(x)=\nabla f_{\ell}(y)=\left(u_{1}, u_{2}\right)\right] \leq C d_{\mathbb{S}^{2}}^{2}(x, y),
$$

and combining together (4.3) and (4.4), we have that

$$
K_{\ell}\left(x, y ; u_{1}, u_{2}\right) \leq \mathrm{const} \text { for all }(x, y) \in \mathbb{S}^{2} \times \mathbb{S}^{2},
$$

whence the result is established.

\section{Proof of Theorem 1}

5.1. Cholesky decomposition. Let us now write $\sigma_{\ell}(x)$ for the $5 \times 5$ covariance matrix of the Gaussian random vector $\left(\nabla f_{\ell}(x), \operatorname{vec}\left(\nabla^{2} f_{\ell}(x)\right)\right)$, i.e. the $5 \times 1$ vector that includes the gradient and the Hessian components of interest. We evaluate the covariance matrix $\sigma_{\ell}(x)$ at any point $x \in \mathbb{S}^{2}$, and we write it in the partitioned form

$$
\sigma_{\ell}(x)_{5 \times 5}=\left(\begin{array}{cc}
a_{\ell}(x) & b_{\ell}(x) \\
b_{\ell}^{T}(x) & c_{\ell}(x)
\end{array}\right),
$$

where as before the superscript $T$ denotes transposition, and

$$
\begin{gathered}
a_{\ell}(x)=\left(\begin{array}{cc}
\frac{\lambda_{\ell}}{2} & 0 \\
0 & \frac{\lambda_{\ell}}{2}
\end{array}\right), \quad b_{\ell}(x)=\left(\begin{array}{ccc}
0 & 0 & 0 \\
0 & 0 & 0
\end{array}\right), \\
c_{\ell}(x)=\frac{\lambda_{\ell}^{2}}{8}\left(\begin{array}{ccc}
3-\frac{2}{\lambda_{\ell}} & 0 & 1+\frac{2}{\lambda_{\ell}} \\
0 & 1-\frac{2}{\lambda_{\ell}} & 0 \\
1+\frac{2}{\lambda_{\ell}} & 0 & 3-\frac{2}{\lambda_{\ell}}
\end{array}\right) .
\end{gathered}
$$

We follow here the same argument as in [10; in particular, we recall that the Cholesky decomposition of a Hermitian positive-definite matrix $A$ takes the form $A=\Lambda \Lambda^{T}$, where $\Lambda$ is a lower triangular matrix with real and positive diagonal entries, and $\Lambda^{T}$ denotes the conjugate transpose of $\Lambda$. It is well-known that every Hermitian positive-definite matrix (and thus also every real-valued symmetric positive-definite matrix) admits a unique Cholesky decomposition.

By an explicit computation, it is then possible to show that the Cholesky decomposition of $\sigma_{\ell}$ takes the form $\sigma_{\ell}=\Lambda_{\ell} \Lambda_{\ell}^{T}$, where

$$
\Lambda_{\ell}=\left(\begin{array}{ccccc}
\frac{\sqrt{\lambda_{\ell}}}{\sqrt{2}} & 0 & 0 & 0 & 0 \\
0 & \frac{\sqrt{\lambda_{\ell}}}{\sqrt{2}} & 0 & 0 & 0 \\
0 & 0 & \frac{\sqrt{\lambda_{\ell}} \sqrt{3 \lambda_{\ell}-2}}{2 \sqrt{2}} & 0 & 0 \\
0 & 0 & 0 & \frac{\sqrt{\lambda_{\ell}} \sqrt{\lambda_{\ell}-2}}{2 \sqrt{2}} & 0 \\
0 & 0 & \frac{\sqrt{\lambda_{\ell}}\left(\lambda_{\ell}+2\right)}{2 \sqrt{2} \sqrt{3 \lambda_{\ell}-2}} & 0 & \frac{\lambda_{\ell} \sqrt{\lambda_{\ell}-2}}{\sqrt{3 \lambda_{\ell}-2}}
\end{array}\right)=:\left(\begin{array}{ccccc}
\lambda_{1} & 0 & 0 & 0 & 0 \\
0 & \lambda_{1} & 0 & 0 & 0 \\
0 & 0 & \lambda_{3} & 0 & 0 \\
0 & 0 & 0 & \lambda_{4} & 0 \\
0 & 0 & \lambda_{2} & 0 & \lambda_{5}
\end{array}\right) ;
$$

in the last expression, for notational simplicity we have omitted the dependence of the $\lambda_{i} \mathrm{~s}$ on $\ell$. The matrix is block diagonal, because under isotropy the gradient components are independent from the Hessian when evaluated at the same point. We can hence define a 5-dimensional standard Gaussian vector $Y(x)=\left(Y_{1}(x), Y_{2}(x), Y_{3}(x), Y_{4}(x), Y_{5}(x)\right)$ with independent components such that

$$
\begin{gathered}
\left(\nabla f_{\ell}(x), \operatorname{vec}\left(\nabla^{2} f_{\ell}(x)\right)\right)=\Lambda_{\ell} Y(x) \\
=\left(\lambda_{1} Y_{1}(x), \lambda_{1} Y_{2}(x), \lambda_{3} Y_{3}(x), \lambda_{4} Y_{4}(x), \lambda_{5} Y_{5}(x)+\lambda_{2} Y_{3}(x)\right) .
\end{gathered}
$$

So we have

$$
\begin{aligned}
\mathcal{N}_{\ell, \varepsilon}^{c}(I) & =\int_{\mathbb{S}^{2}}\left|\operatorname{det} H_{f_{\ell}}(x)\right| \mathbb{I}_{\left\{\left(\partial_{11, x} f_{\ell}(x)+\partial_{22, x} f_{\ell}(x)\right) / \lambda_{\ell} \in I\right\}} \delta_{\varepsilon}\left(\nabla f_{\ell}(x)\right) d x \\
& =\int_{\mathbb{S}^{2}}\left|\lambda_{3} Y_{3}(x)\left(\lambda_{5} Y_{5}(x)+\lambda_{2} Y_{3}(x)\right)-\left(\lambda_{4} Y_{4}(x)\right)^{2}\right| \mathbb{I}_{\left\{\frac{\lambda_{2}+\lambda_{3}}{\lambda} Y_{3}+\frac{\lambda_{5}}{\lambda} Y_{5} \in I\right\}} \delta_{\varepsilon}\left(\lambda_{1} Y_{1}(x), \lambda_{1} Y_{2}(x)\right) d x
\end{aligned}
$$




$$
=\frac{\lambda^{2}}{\lambda_{1}^{2}} \int_{\mathbb{S}^{2}}\left|\frac{\lambda_{3} \lambda_{5}}{\lambda^{2}} Y_{3}(x) Y_{5}(x)+\frac{\lambda_{2} \lambda_{3}}{\lambda^{2}} Y_{3}^{2}(x)-\frac{\lambda_{4}^{2}}{\lambda^{2}} Y_{4}^{2}(x)\right| \mathbb{I}_{\left\{\frac{\lambda_{2}+\lambda_{3}}{\lambda} Y_{3}+\frac{\lambda_{5}}{\lambda} Y_{5} \in I\right\}} \delta_{\varepsilon}\left(Y_{1}(x), Y_{2}(x)\right) d x .
$$

5.2. Second order chaotic component. Following the same argument as in [10, it can be shown that the second order chaotic component of the number of critical points is given by

$$
\mathcal{N}_{\ell ; I}^{c}[2]=\frac{\lambda^{2}}{\lambda_{1}^{2}}\left[\sum_{i<j} h_{i j}(\ell ; I) \int_{\mathbb{S}^{2}} Y_{i}(x) Y_{j}(x) d x+\frac{1}{2} \sum_{i=1}^{5} k_{i}(\ell ; I) \int_{\mathbb{S}^{2}} H_{2}\left(Y_{i}(x)\right) d x\right],
$$

where

$$
\begin{aligned}
& h_{i j}(\ell ; I)=\lim _{\varepsilon \rightarrow 0} \mathbb{E}\left[\left|\frac{\lambda_{3} \lambda_{5}}{\lambda^{2}} Y_{3} Y_{5}+\frac{\lambda_{2} \lambda_{3}}{\lambda^{2}} Y_{3}^{2}-\frac{\lambda_{4}^{2}}{\lambda^{2}} Y_{4}^{2}\right| Y_{i} Y_{j} \mathbb{I}_{\left\{\frac{\lambda_{2}+\lambda_{3}}{\lambda} Y_{3}+\frac{\lambda_{5}}{\lambda} Y_{5} \in I\right\}} \delta_{\varepsilon}\left(Y_{1}, Y_{2}\right)\right], \\
& k_{i}(\ell ; I)=\lim _{\varepsilon \rightarrow 0} \mathbb{E}\left[\left|\frac{\lambda_{3} \lambda_{5}}{\lambda^{2}} Y_{3} Y_{5}+\frac{\lambda_{2} \lambda_{3}}{\lambda^{2}} Y_{3}^{2}-\frac{\lambda_{4}^{2}}{\lambda^{2}} Y_{4}^{2}\right| H_{2}\left(Y_{i}\right) \mathbb{I}_{\left\{\frac{\lambda_{2}+\lambda_{3}}{\lambda} Y_{3}+\frac{\lambda_{5}}{\lambda} Y_{5} \in I\right\}} \delta_{\varepsilon}\left(Y_{1}, Y_{2}\right)\right] .
\end{aligned}
$$

Note, however, that the computation of projection coefficients here is different (and considerably more complicated) than in 10, due to the presence of the absolute value in the previous formulae (5.15.2), which makes the evaluation of exact moments much more challenging. In particular, the computation of the projection coefficients $h_{i j}(.,),. k_{i}(.,$.$) is collected in two Lemmas below.$

Lemma 6. For every $I \subset \mathbb{R}$, it holds that $h_{1 j}(\ell ; I), h_{2 j}(\ell ; I)=0$, for $j=1, \ldots 5$.

Proof. The statement follows immediately from

$$
\lim _{\varepsilon \rightarrow 0} \mathbb{E}\left[H_{1}(Y) \delta_{\varepsilon}(Y)\right]=0
$$

Lemma 7. For every $I \subset \mathbb{R}$, it holds that $h_{34}(\ell ; I), h_{45}(\ell ; I)=O\left(\ell^{-1}\right)$.

Proof. Note that

$$
\lim _{\varepsilon \rightarrow 0} \mathbb{E}\left[H_{0}(Y) \delta_{\varepsilon}(Y)\right]=\frac{1}{\sqrt{2 \pi}} .
$$

To prove that $h_{34}(\ell ; I)=O\left(\ell^{-1}\right)$ we first apply (5.3) to obtain

$$
\begin{aligned}
h_{34}(\ell ; I) & =\lim _{\varepsilon \rightarrow 0} \mathbb{E}\left[\left|\frac{\lambda_{3} \lambda_{5}}{\lambda^{2}} Y_{3} Y_{5}+\frac{\lambda_{2} \lambda_{3}}{\lambda^{2}} Y_{3}^{2}-\frac{\lambda_{4}^{2}}{\lambda^{2}} Y_{4}^{2}\right| Y_{4} Y_{3} \mathbb{I}_{\left\{\frac{\lambda_{2}+\lambda_{3}}{\lambda} Y_{3}+\frac{\lambda_{5}}{\lambda} Y_{5} \in I\right\}}\right] \mathbb{E}\left[\delta_{\varepsilon}\left(Y_{1}, Y_{2}\right)\right] \\
& =\frac{1}{2 \pi} \mathbb{E}\left[\left|\frac{\lambda_{3} \lambda_{5}}{\lambda^{2}} Y_{3} Y_{5}+\frac{\lambda_{2} \lambda_{3}}{\lambda^{2}} Y_{3}^{2}-\frac{\lambda_{4}^{2}}{\lambda^{2}} Y_{4}^{2}\right| Y_{4} Y_{3} \mathbb{I}_{\left\{\frac{\lambda_{2}+\lambda_{3}}{\lambda} Y_{3}+\frac{\lambda_{5}}{\lambda} Y_{5} \in I\right\}}\right] \\
& =\frac{1}{2 \pi} \mathbb{E}\left[\left|\frac{1}{\sqrt{8}} Y_{3} Y_{5}+\frac{1}{8} Y_{3}^{2}-\frac{1}{8} Y_{4}^{2}\right| Y_{4} Y_{3} \mathbb{I}_{\left\{\frac{\sqrt{2}}{\sqrt{3}} Y_{3}+\frac{1}{\sqrt{3}} Y_{5} \in I\right\}}\right]+O\left(\ell^{-1}\right),
\end{aligned}
$$

then we use the following transformation

$$
Y_{3}=\frac{1}{\sqrt{3}} Z_{1}, \quad Y_{4}=Z_{2}, \quad Y_{5}=\frac{\sqrt{3}}{\sqrt{8}} Z_{3}-\frac{1}{\sqrt{3} \sqrt{8}} Z_{1}
$$

where $Z=\left(Z_{1}, Z_{2}, Z_{3}\right)$ is a centred jointly Gaussian random vector with covariance matrix

$$
\left(\begin{array}{lll}
3 & 0 & 1 \\
0 & 1 & 0 \\
1 & 0 & 3
\end{array}\right)
$$

We can rewrite $h_{34}(\ell ; I)$ as follows

$$
\begin{aligned}
h_{34}(\ell ; I) & =\frac{1}{2 \pi} \frac{1}{8 \sqrt{3}} \mathbb{E}\left[\left|Z_{1} Z_{3}-Z_{2}^{2}\right| Z_{1} Z_{2} \mathbb{I}_{\left\{\frac{1}{\sqrt{8}}\left(Z_{1}+Z_{3}\right) \in I\right\}}\right]+O\left(\ell^{-1}\right) \\
& =\frac{1}{2 \pi} \frac{1}{8 \sqrt{3}} \int_{I} d t \mathbb{E}\left[\left|Z_{1} Z_{3}-Z_{2}^{2}\right| Z_{1} Z_{2} \mathbb{I}_{\left\{Z_{1}+Z_{3}=t \sqrt{8}\right\}}\right]+O\left(\ell^{-1}\right) .
\end{aligned}
$$

We introduce the transformation $W_{1}=Z_{1}, W_{2}=Z_{2}, W_{3}=Z_{1}+Z_{3}$ so that

$$
\mathbb{E}\left[\left|Z_{1} Z_{3}-Z_{2}^{2}\right| Z_{1} Z_{2} \mathbb{I}_{\left\{Z_{1}+Z_{3}=t \sqrt{8}\right\}}\right]=\mathbb{E}\left[\left|W_{1}\left(W_{3}-W_{1}\right)-W_{2}^{2}\right| W_{1} W_{2} \mathbb{I}_{\left\{W_{3}=t \sqrt{8}\right\}}\right]
$$




$$
=\phi_{W_{3}}(t \sqrt{8}) \mathbb{E}\left[\left|W_{1}\left(W_{3}-W_{1}\right)-W_{2}^{2}\right| W_{1} W_{2} \mid W_{3}=t \sqrt{8}\right],
$$

where $\phi_{W_{3}}$ is the density function of $W_{3}$. In [7] we proved that the bivariate conditioned Gaussian vector $\left(W_{1}, W_{2}\right) \mid W_{3}=\sqrt{8} t$ is distributed as $\left(X_{1}+\sqrt{2} t, X_{2}\right)$ where $\left(X_{1}, X_{2}\right)$ are independent standard Gaussian, and hence

$$
\mathbb{E}\left[\left|W_{1}\left(W_{3}-W_{1}\right)-W_{2}^{2}\right| W_{1} W_{2} \mid W_{3}=t \sqrt{8}\right]=\mathbb{E}\left[\left|2 t^{2}-X_{1}^{2}-X_{2}^{2}\right|\left(X_{1}+\sqrt{2} t\right) X_{2}\right] .
$$

We finally note that by symmetry

$$
\mathbb{E}\left[\left|2 t^{2}-X_{1}^{2}-X_{2}^{2}\right| X_{2}\right]=0 \text { and } \mathbb{E}\left[\left|2 t^{2}-X_{1}^{2}-X_{2}^{2}\right| X_{1} X_{2}\right]=0 .
$$

In the same way we see that $h_{45}(\ell ; I)=O\left(\ell^{-1}\right)$.

For convenience, we recall now in the following Lemma the results in [10, Proposition 6:

Lemma 8. We have

$$
\int_{\mathbb{S}^{2}} Y_{3}(x) Y_{5}(x) d x=4 \pi \frac{\sqrt{2}}{3} \sum_{m=-\ell}^{\ell}\left\{\left|a_{\ell m}\right|^{2}-1\right\}\left[-\frac{1}{\ell}+\frac{3 m}{\ell^{2}}-\frac{2 m^{3}}{\ell^{4}}\right]+r_{0}(\ell),
$$

and moreover

$$
\begin{aligned}
& \int_{\mathbb{S}^{2}} H_{2}\left(Y_{1}(x)\right) d x=4 \pi \sum_{m=-\ell}^{\ell}\left\{\left|a_{\ell m}\right|^{2}-1\right\}\left[\frac{1}{\ell}-\frac{m}{\ell^{2}}\right]+r_{1}(\ell), \\
& \int_{\mathbb{S}^{2}} H_{2}\left(Y_{2}(x)\right) d x=4 \pi \sum_{m=-\ell}^{\ell}\left\{\left|a_{\ell m}\right|^{2}-1\right\} \frac{m}{\ell^{2}}+r_{2}(\ell), \\
& \int_{\mathbb{S}^{2}} H_{2}\left(Y_{3}(x)\right) d x=4 \pi \sum_{m=-\ell}^{\ell}\left\{\left|a_{\ell m}\right|^{2}-1\right\}\left[\frac{4}{3 \ell}-\frac{2 m}{\ell^{2}}+\frac{2 m^{3}}{3 \ell^{4}}\right]+r_{3}(\ell), \\
& \int_{\mathbb{S}^{2}} H_{2}\left(Y_{4}(x)\right) d x=4 \pi \times 2 \sum_{m=-\ell}^{\ell}\left\{\left|a_{\ell m}\right|^{2}-1\right\}\left[\frac{m}{\ell^{2}}-\frac{m^{3}}{\ell^{4}}\right]+r_{4}(\ell), \\
& \int_{\mathbb{S}^{2}} H_{2}\left(Y_{5}(x)\right) d x=4 \pi \times \frac{1}{6} \sum_{m=-\ell}^{\ell}\left\{\left|a_{\ell m}\right|^{2}-1\right\}\left[\frac{1}{\ell}+\frac{8 m^{3}}{\ell^{4}}\right]+r_{5}(\ell),
\end{aligned}
$$

where $\sqrt{\mathbb{E}\left[r_{i}(\ell)\right]^{2}}=O\left(\ell^{-1}\right)$, for all $i=0, \ldots, 5$.

From Lemma 8 we deduce the following:

Lemma 9. We have that

$$
\int_{\mathbb{S}^{2}} Y_{3}(x) Y_{4}(x) d x=r_{6}(\ell), \quad \int_{\mathbb{S}^{2}} Y_{4}(x) Y_{5}(x) d x=r_{7}(\ell),
$$

where $\sqrt{\mathbb{E}\left[r_{i}(\ell)\right]^{2}}=O(1)$, for $i=6,7$.

Proof. Let, for $i, j, k, l=1,2$,

$$
I_{i j, k l}(\ell)=\int_{\mathbb{S}^{2}} \partial_{i j, x} f_{\ell}(x) \partial_{k l, x} f_{\ell}(x) d x,
$$

and

$$
I_{00}(\ell)=\int_{\mathbb{S}^{2}} f_{\ell}^{2}(x) d x, \quad I_{0,22}(\ell)=\int_{\mathbb{S}^{2}} f_{\ell}(x) \partial_{22, x} f_{\ell}(x) d x .
$$

We immediately see that

$$
\int_{\mathbb{S}^{2}} Y_{3}(x) Y_{4}(x) d x=\frac{1}{\lambda_{3} \lambda_{4}} \int_{\mathbb{S}^{2}} \partial_{11, x} f_{\ell}(x) \partial_{21, x} f_{\ell}(x) d x=\frac{1}{\lambda_{3} \lambda_{4}} I_{11,12}(\ell),
$$

and

$$
\begin{aligned}
\int_{\mathbb{S}^{2}} Y_{4}(x) Y_{5}(x) d x & =\frac{1}{\lambda_{4} \lambda_{5}} \int_{\mathbb{S}^{2}} \partial_{12, x} f_{\ell}(x)\left(\partial_{22, x} f_{\ell}(x)-\frac{\lambda_{2}}{\lambda_{3}} \partial_{11, x} f_{\ell}(x)\right) d x \\
& =\frac{1}{\lambda_{4} \lambda_{5}} I_{12,22}(\ell)-\frac{\lambda_{2}}{\lambda_{3} \lambda_{4} \lambda_{5}} I_{11,12}(\ell) .
\end{aligned}
$$


Applying Cauchy-Schwarz we also have

$$
I_{11,12}(\ell) \leq \sqrt{I_{11,11}(\ell) I_{12,12}(\ell)}, \quad I_{12,22}(\ell) \leq \sqrt{I_{12,12}(\ell) I_{22,22}(\ell)} ;
$$

where

The results in Lemma 8

$$
I_{11,11}(\ell)=\lambda_{\ell}^{2} I_{00}(\ell)+I_{22,22}(\ell)+2 \lambda_{\ell} I_{0,22}(\ell) .
$$

$$
\begin{aligned}
& I_{00}(\ell)=\frac{1}{2 \ell+1} \sum_{m=-\ell}^{\ell}\left|a_{\ell m}\right|^{2}, \\
& I_{0,22}(\ell)=-a_{\ell 0}^{2} \frac{\ell}{2 \ell+1}+\sum_{m>0}\left|a_{\ell m}\right|^{2}\left(\frac{1}{2 \ell+1}-m\right), \\
& I_{12,12}(\ell)=\sum_{m=-\ell}^{\ell}\left|a_{\ell m}\right|^{2} m\left\{\frac{\lambda_{\ell}-1-m^{2}}{4}\right\}, \\
& I_{22,22}(\ell)=\frac{a_{\ell 0}^{2}}{2}\left(\ell^{2}-\frac{\ell}{2 \ell+1}\right)+\frac{1}{2} \sum_{m>0}\left|a_{\ell m}\right|^{2}\left\{-\frac{4 \lambda_{\ell}}{2 \ell+1}+m+\lambda m+m^{3}\right\} .
\end{aligned}
$$

immediately imply that

$$
\int_{\mathbb{S}^{2}} Y_{3}(x) Y_{4}(x) d x=r_{6}(\ell), \quad \int_{\mathbb{S}^{2}} Y_{5}(x) Y_{4}(x) d x=r_{7}(\ell) ;
$$

where $\sqrt{\mathbb{E}\left[r_{i}(\ell)\right]^{2}}=O(1)$, for $i=6,7$.

In view of Lemmas 6, 7 , 9 we have

$$
\mathcal{N}_{\ell ; I}^{c}[2]=\frac{\lambda^{2}}{\lambda_{1}^{2}}\left[h_{35}(\ell ; I) \int_{\mathbb{S}^{2}} Y_{3}(x) Y_{5}(x) d x+\frac{1}{2} \sum_{i=1}^{5} k_{i}(\ell ; I) \int_{\mathbb{S}^{2}} H_{2}\left(Y_{i}(x)\right) d x\right]+R_{3}(\ell),
$$

where $\frac{\lambda^{2}}{\lambda_{1}^{2}}=2 \lambda_{\ell}$ and $\sqrt{\mathbb{E}\left[R_{3}(\ell)\right]^{2}}=O(\ell)$ uniformly over $I$. Our next step is now to rewrite $\mathcal{N}_{\ell ; I}^{c}[2]$ rearranging the projection coefficients to make their computations more manageable. Indeed, recalling their definitions in 5.1 5.2 and exploting the analytic expressions in Lemma 8, some tedious but straightforward algebra yields that

$$
\mathcal{N}_{\ell ; I}^{c}[2]=2 \lambda_{\ell} \frac{2}{\ell} \sum_{m=-\ell}^{\ell}\left\{\left|a_{\ell m}\right|^{2}-1\right\}\left[M_{0}+\frac{m}{\ell} M_{1}+\frac{1}{3} \frac{m^{3}}{\ell^{3}} M_{2}\right]+R_{3}(\ell),
$$

where

$$
\begin{aligned}
& M_{0}(I):=\mathbb{E}\left[\left|\frac{1}{\sqrt{8}} Y_{3} Y_{5}+\frac{1}{8} Y_{3}^{2}-\frac{1}{8} Y_{4}^{2}\right|\left(-\frac{5}{4}+\frac{2}{3} Y_{3}^{2}-\frac{\sqrt{2}}{3} Y_{3} Y_{5}+\frac{1}{12} Y_{5}^{2}\right) \mathbb{I}_{\left\{\frac{\sqrt{2}}{\sqrt{3}} Y_{3}+\frac{1}{\sqrt{3}} Y_{5} \in I\right\}}\right], \\
& M_{1}(I):=\mathbb{E}\left[\left|\frac{1}{\sqrt{8}} Y_{3} Y_{5}+\frac{1}{8} Y_{3}^{2}-\frac{1}{8} Y_{4}^{2}\right|\left(-Y_{3}^{2}+Y_{4}^{2}+\sqrt{2} Y_{3} Y_{5}\right) \mathbb{I}_{\left\{\frac{\sqrt{2}}{\sqrt{3}} Y_{3}+\frac{1}{\sqrt{3}} Y_{5} \in I\right\}}\right], \\
& M_{2}(I):=\mathbb{E}\left[\left|\frac{1}{\sqrt{8}} Y_{3} Y_{5}+\frac{1}{8} Y_{3}^{2}-\frac{1}{8} Y_{4}^{2}\right|\left(Y_{3}^{2}-3 Y_{4}^{2}-2 \sqrt{2} Y_{3} Y_{5}+2 Y_{5}^{2}\right) \mathbb{I}_{\left\{\frac{\sqrt{2}}{\sqrt{3}} Y_{3}+\frac{1}{\sqrt{3}} Y_{5} \in I\right\}}\right] .
\end{aligned}
$$

It is then possible to prove that:

Lemma 10. For all $I \subset \mathbb{R}$, we have $M_{1}(I)=M_{2}(I)=0$ and

$$
M_{0}(I)=\frac{1}{8} \int_{I} p_{3}^{c}(t) d t,
$$

where $p_{3}^{c}($.$) is defined in (1.6).$

Proof. Note that after the transformation

we immediately obtain

$$
Y_{3}=\frac{1}{\sqrt{3}} Z_{1}, \quad Y_{4}=Z_{2}, \quad Y_{5}=\frac{\sqrt{3}}{\sqrt{8}} Z_{3}-\frac{1}{\sqrt{3} \sqrt{8}} Z_{1},
$$

$$
M_{0}(I)=\frac{1}{8} \frac{1}{32} \mathbb{E}\left[\left|Z_{1} Z_{3}-Z_{2}^{2}\right|\left[-40+\left(-3 Z_{1}+Z_{3}\right)^{2}\right] \mathbb{I}_{\left\{\frac{1}{\sqrt{8}}\left(Z_{1}+Z_{3}\right) \in I\right\}}\right],
$$


and

since

$$
M_{1}(I)=\frac{1}{3} M_{2}=\frac{1}{8} \frac{1}{2} \mathbb{E}\left[\left|Z_{1} Z_{3}-Z_{2}^{2}\right|\left[2 Z_{2}^{2}+Z_{1}\left(Z_{3}-Z_{1}\right)\right] \mathbb{I}_{\left\{\frac{1}{\sqrt{8}}\left(Z_{1}+Z_{3}\right) \in I\right\}}\right],
$$

$$
\begin{aligned}
& \mathbb{E}\left[\left|Z_{1} Z_{3}-Z_{2}^{2}\right|\left[4 Z_{2}^{2}+2 Z_{1} Z_{3}-2 Z_{1}^{2}\right] \mathbb{I}_{\left\{\frac{1}{\sqrt{8}}\left(Z_{1}+Z_{3}\right) \in I\right\}}\right] \\
& =\mathbb{E}\left[\left|Z_{1} Z_{3}-Z_{2}^{2}\right|\left[-4 Z_{2}^{2}+Z_{1}^{2}+Z_{3}^{2}-2 Z_{1} Z_{3}\right] \mathbb{I}_{\left\{\frac{1}{\sqrt{8}}\left(Z_{1}+Z_{3}\right) \in I\right\}}\right] .
\end{aligned}
$$

We write $W_{1}=Z_{1}, W_{2}=Z_{2}, W_{3}=Z_{1}+Z_{3}$, i.e. $Z_{3}=W_{3}-W_{1}$ so that

$$
\begin{aligned}
M_{1}(I) & =\frac{1}{8} \frac{1}{4} \mathbb{E}\left[\left|W_{1}\left(W_{3}-W_{1}\right)-W_{2}^{2}\right|\left[4 W_{2}^{2}+2 W_{1}\left(W_{3}-W_{1}\right)-2 W_{1}^{2}\right] \mathbb{I}_{\left\{\frac{1}{\sqrt{8}} W_{3} \in I\right\}}\right] \\
& =\frac{1}{8} \frac{1}{4} \int_{I} \mathbb{E}\left[\left|W_{1} W_{3}-W_{1}^{2}-W_{2}^{2}\right|\left[4 W_{2}^{2}+2 W_{1} W_{3}-4 W_{1}^{2}\right] \mid W_{3}=\sqrt{8} t\right] \phi_{W_{3}}(\sqrt{8} t) d t
\end{aligned}
$$

where

$$
\begin{aligned}
& \mathbb{E}\left[\left|W_{1} W_{3}-W_{1}^{2}-W_{2}^{2}\right|\left[4 W_{2}^{2}+2 W_{1} W_{3}-4 W_{1}^{2}\right] \mid W_{3}=\sqrt{8} t\right] \\
& =\mathbb{E}\left[\left|\sqrt{8} t W_{1}-W_{1}^{2}-W_{2}^{2}\right|\left[4 W_{2}^{2}+2 \sqrt{8} t W_{1}-4 W_{1}^{2}\right] \mid W_{3}=\sqrt{8} t\right]
\end{aligned}
$$

since we know that $\left(W_{1}, W_{2}\right) \mid W_{3}=\sqrt{8} t$ is distributed as $\left(X_{1}+\sqrt{2} t, X_{2}\right)$ where $\left(X_{1}, X_{2}\right)$ are independent standard Gaussian, we have

$$
\begin{aligned}
& \mathbb{E}\left[\left|\sqrt{8} t\left(X_{1}+\sqrt{2} t\right)-\left(X_{1}+\sqrt{2} t\right)^{2}-X_{2}^{2}\right|\left[4 X_{2}^{2}+2 \sqrt{8} t\left(X_{1}+\sqrt{2} t\right)-4\left(X_{1}+\sqrt{2} t\right)^{2}\right]\right] \\
& =\mathbb{E}\left[\left|-X_{1}^{2}-X_{2}^{2}+2 t^{2}\right|\left[4 X_{2}^{2}+2 \sqrt{8} t\left(X_{1}+\sqrt{2} t\right)-4\left(X_{1}+\sqrt{2} t\right)^{2}\right]\right] \\
& =\mathbb{E}\left[\left|-X_{1}^{2}-X_{2}^{2}+2 t^{2}\right|\left[4 X_{2}^{2}-4 X_{1}^{2}-4 \sqrt{2} t X_{1}\right]\right] \\
& =-4 \sqrt{2} t \mathbb{E}\left[\left|-X_{1}^{2}-X_{2}^{2}+2 t^{2}\right| X_{1}\right]=0
\end{aligned}
$$

so that the first part of the Lemma is proved. Also, we have that

$$
M_{0}(I)=\frac{1}{256}\left[-40 \mathcal{I}_{I, 0}+\mathcal{I}_{I, 2}\right]
$$

with

$$
\mathcal{I}_{I, 0}=\mathbb{E}\left[\left|Z_{1} Z_{3}-Z_{2}^{2}\right| \mathbb{I}_{\left\{\frac{1}{\sqrt{8}}\left(Z_{1}+Z_{3}\right) \in I\right\}}\right], \mathcal{I}_{I, 2}=\mathbb{E}\left[\left|Z_{1} Z_{3}-Z_{2}^{2}\right|\left(Z_{1}-3 Z_{3}\right)^{2} \mathbb{I}_{\left\{\frac{1}{\sqrt{8}}\left(Z_{1}+Z_{3}\right) \in I\right\}}\right] .
$$

The final expression for $\mathcal{N}_{\ell ; I}^{c}[2]$ given in Theorem 1 immediately follows by noting that (using the same notation as in [7])

where

$$
\mathcal{I}_{I, 0}=\int_{I} p_{0}^{c}(t) d t, \quad \mathcal{I}_{I, 2}=8 \int_{I} p_{2}^{c}(t) d t
$$

Hence

$$
p_{0}^{c}(t)=\sqrt{\frac{2}{\pi}}\left[2 e^{-t^{2}}+t^{2}-1\right] e^{-\frac{t^{2}}{2}}, \quad p_{2}^{c}(t)=\sqrt{\frac{2}{\pi}}\left[-4+t^{2}+t^{4}+e^{-t^{2}} 2\left(4+3 t^{2}\right)\right] e^{-\frac{t^{2}}{2}} .
$$

$$
M_{0}(I)=\frac{1}{8}\left\{\int_{I}\left(-\frac{5}{4} p_{0}^{c}(t)+\frac{1}{4} p_{2}^{c}(t)\right) d t\right\}=\frac{1}{8} \int_{I} p_{3}^{c}(t) d t,
$$

as claimed.

We have hence obtained

$$
\begin{aligned}
\mathcal{N}_{\ell ; I}^{c}[2] & =2 \lambda_{\ell} \frac{2}{\ell} M_{0}(I) \sum_{m=-\ell}^{\ell}\left\{\left|a_{\ell m}\right|^{2}-1\right\}+R_{3}(\ell) \\
& =\ell(\ell+1) \frac{4}{\ell} \frac{1}{8}\left\{\int_{I} p_{3}^{c}(t) d t\right\} \sum_{m=-\ell}^{\ell}\left\{\left|a_{\ell m}\right|^{2}-1\right\}+R_{3}(\ell), \\
& =\frac{(\ell+1)}{2}\left\{\int_{I} p_{3}^{c}(t) d t\right\} \sum_{m=-\ell}^{\ell}\left\{\left|a_{\ell m}\right|^{2}-1\right\}+R_{3}(\ell) .
\end{aligned}
$$


Finally, to complete the proof we need only show that

$$
\lim _{\ell \rightarrow \infty} \frac{\operatorname{Var}\left\{\mathcal{N}_{\ell ; I}^{c}\right\}}{\operatorname{Var}\left\{\mathcal{N}_{\ell ; I}^{c}[2]\right\}}=1+\lim _{\ell \rightarrow \infty} \frac{\sum_{q \geq 3} \operatorname{Var}\left\{\mathcal{N}_{\ell ; I}^{c}[q]\right\}}{\operatorname{Var}\left\{\mathcal{N}_{\ell ; I}^{c}[2]\right\}}=0 ;
$$

indeed we have that

$$
\begin{aligned}
\operatorname{Var}\left((\ell+1) \frac{1}{2} \int_{I} p_{3}^{c}(t) d t \sum_{m=-\ell}^{\ell}\left\{\left|a_{\ell m}\right|^{2}-1\right\}\right) & =\frac{(\ell+1)^{2}}{4}\left\{\int_{I} p_{3}^{c}(t) d t\right\}^{2} \operatorname{Var}\left(\sum_{m=-\ell}^{\ell}\left\{\left|a_{\ell m}\right|^{2}-1\right\}\right) \\
& =\frac{(\ell+1)^{2}}{4}\left\{\int_{I} p_{3}^{c}(t) d t\right\}^{2} 2(2 \ell+1) \\
& =\ell^{3}\left\{\int_{I} p_{3}^{c}(t) d t\right\}^{2}+O\left(\ell^{2}\right),
\end{aligned}
$$

where the last term on the right hand side is the variance for $\mathcal{N}_{\ell ; I}^{c}$ obtained in [7], as claimed.

\section{REFERENCES}

[1] R.J.Adler, J. E.Taylor (2007) Random Fields and Geometry. Springer Monographs in Mathematics, Springer

[2] R.J.Adler, J.E.Taylor (2011) Topological Complexity of Smooth Random Functions. Lect.39th Prob.School Saint-Flour, Springer

[3] J.-M.Azaïs, M.Wschebor (2009) Level Sets and Extrema of Random Processes and Fields. John Wiley \& Sons

[4] J.Benatar, D.Marinucci, I.Wigman (2017) Planck-scale distribution of nodal length of arithmetic random waves, preprint, arXiv:1710.06153

[5] M.V.Berry (1977) Regular and irregular semiclassical wavefunctions, Journal of Physics A, 10, 12, 2083-2091

[6] J.Buckley, I.Wigman (2016) On the number of nodal domains of toral eigenfunctions, Annalés Henri Poincaré 17, no. 11, 3027-3062.

[7] V.Cammarota, D.Marinucci, I.Wigman (2016) On the distribution of the critical values of random spherical harmonics, Journal of Geometric Analysis, 4, 3252-3324

[8] V.Cammarota, D.Marinucci, I.Wigman (2016) Fluctuations of the Euler-Poincaré characteristic for random spherical harmonics, Proceedings of the American Mathematical Society, 11, 4759-4775

[9] V.Cammarota, I.Wigman (2017) Fluctuations of the total number of critical points of random spherical harmonics, Stochastic Processes and their Applications, 127, no. 12, 3825-3869

[10] V.Cammarota, D.Marinucci, (2018) A Quantitative Central Limit Theorem for the Euler-Poincaré Characteristic of Random Spherical Eigenfunctions, Annals of Probability, in press, arXiv:1603.09588

[11] I.Chavel (2006) Riemannian Geometry. A Modern Introduction. Cambridge University Press

[12] D.Cheng, Y.Xiao (2016) Excursion probability of Gaussian random fields on sphere, Bernoulli, 22,2, 1113-1130

[13] D.Cheng, Y.Xiao (2016) The mean Euler characteristic and excursion probability of Gaussian random fields, Annals of Applied Probability, 26, 2, 722-759

[14] F. Dalmao, I. Nourdin, G. Peccati, M. Rossi (2016), Phase singularities in complex arithmetic random waves, Preprint, arXiv:1608.05631

[15] H.Dehling and M.Taqqu (1989) The empirical process of some long-range dependent sequences with an application to Ustatistics, Annals of Statistics 17, no. 4,1767-1783.

[16] A.Estrade, J.R.Leon (2016) A central limit theorem for the Euler characteristic of a Gaussian excursion set, Annals of Probability, 44, 6, 3849-3878

[17] R.Feng, R.J.Adler (2017) Critical radius and supremum of random spherical harmonics, Annals of Probability, in press, arXiv:1702.02767

[18] A.Granville, I.Wigman (2018) Planck-scale mass equidistribution of toral Laplace eigenfunctions, Communications in Mathematical Physics, 355, no. 2, 767-802.

[19] X.Han (2017) Small scale equidistribution of random eigenbases, Communications in Mathematical Physics, 349, no. 1, 425-440. 58J51

[20] M.Krishnapur, P.Kurlberg, I.Wigman (2013) Nodal length fluctuations for arithmetic random waves, Annals of Mathematics, $177,2,699-737$

[21] N.N.Lebedev (1965) Special Functions and their Applications. Prentice-Hall, Inc., Englewood Cliffs, N.J.

[22] D.Marinucci, G.Peccati (2011) Random Fields on the Sphere: Representations, Limit Theorems and Cosmological Applications, Cambridge University Press

[23] D.Marinucci, G.Peccati, M.Rossi, I.Wigman (2016) Non-universality of nodal length distribution for arithmetic random waves, Geometric and Functional Analysis, 26, 926-960

[24] D.Marinucci, M.Rossi (2015) Stein-Malliavin approximations for nonlinear functionals of random eigenfunctions on $S^{d}$, Journal of Functional Analysis, 268,8, 2379-2420

[25] D.Marinucci, M.Rossi, I.Wigman (2017) The asymptotic equivalence of the sample trispectrum and the nodal length for random spherical harmonics, preprint, arXiv:1705.05747

[26] D.Marinucci, I.Wigman (2011) On the area of excursion sets of spherical Gaussian eigenfunctions, Journal of Mathematical Physics, 52, 9, 093301 
[27] D.Marinucci, I.Wigman (2011). The defect variance of random spherical harmonics, Journal of Physics A-Mathematical And Theoretical, 44, no. 35.

[28] D.Marinucci, I.Wigman (2014) On nonlinear functionals of random spherical eigenfunctions, Communications in Mathematical Physics, 327, no. 3, 849-872

[29] F.Nazarov, M.Sodin (2009) On the number of nodal domains of random spherical harmonics, American Journal of Mathematics, 131,5,1337-1357

[30] I.Nourdin, G.Peccati (2012) Normal Approximations Using Malliavin Calculus: from Stein's Method to Universality, Cambridge University Press

[31] I.Nourdin, G.Peccati, M.Rossi (2017) Nodal statistics of planar random waves, preprint, arXiv:1708.02281

[32] G.Peccati, M.Rossi (2016) Quantitative limit theorems for local functionals of arithmetic random waves, Preprint, arXiv:1702.03765

[33] M.Rossi (2018), Random nodal lengths and Wiener chaos, Preprint, arXiv:1803.09716.

[34] Z. Rudnick, I. Wigman (2016) Nodal intersections for random eigenfunctions on the torus, American Journal of Mathematics, 138, no. 6, 1605-1644.

[35] Z.Rudnick, I.Wigman, N.Yesha (2016) Nodal intersections for random waves on the 3-dimensional torus, Annalés Institut Fourier, 66, no. 6, 2455-2484

[36] I.Wigman, (2009) On the distribution of the nodal sets of random spherical harmonics, Journal of Mathematical Physics, 50, no. $1,013521,44 \mathrm{pp}$.

[37] I.Wigman (2010) Fluctuations of the nodal length of random spherical harmonics, Communications in Mathematical Physics, $298,3,787-831$

\section{Appendix A: Levi-Civita Connection and the Hessian}

We start by recalling that in the usual spherical coordinates $(\theta, \varphi)$ the metric tensor on the tangent plane $T\left(\mathbb{S}^{2}\right)$ is given by

$$
g(\theta, \varphi)=\left[\begin{array}{cc}
1 & 0 \\
0 & \sin ^{2} \theta
\end{array}\right]
$$

The computation of the number of critical points by means of the Kac-Rice formula requires the Hessian of our eigenfunctions; the latter requires the notion of covariant derivatives (i.e., the Levi-Civita connection) on $\mathbb{S}^{2}$. Recall indeed that the Levi-Civita connection is the only application $\nabla: T\left(\mathbb{S}^{2}\right) \times T\left(\mathbb{S}^{2}\right) \rightarrow T\left(\mathbb{S}^{2}\right)$ such that

- $\nabla_{X} Y$ is $C^{\infty}$-linear in $X$, i.e. for $f, g \in C^{\infty}\left(\mathbb{S}^{2}\right)$ and $X, Z \in T\left(\mathbb{S}^{2}\right)$, we have that $\nabla_{f X+g Z} Y=$ $f \nabla_{X} Y+g \nabla_{Z} Y$

- $\nabla_{X} Y$ is $\mathbb{R}$-linear in $Y$, i.e. for $a, b \in \mathbb{R}$ and $Y, Z \in T\left(\mathbb{S}^{2}\right)$, we have that $\nabla_{X}(a Y+b Z)=a \nabla_{X} Y+b \nabla_{X} Z$

- Leibnitz rule holds, i.e. $\nabla_{X} f Y=(X f) Y+f \nabla_{X} Y$

- The operator is compatible with the metric, $X g(Y, Z)=g\left(\nabla_{X} Y, Z\right)+g\left(Y, \nabla_{X} Z\right)$

- The operator is torsion-free, i.e. $X Y-Y X=\nabla_{X} Y-\nabla_{Y} X$

See e.g. [1] Chapter 7 for more discussion and details. In coordinates, the action of the Levi-Civita connection can be obtained by means of the so-called Christoffel symbols, see e.g. 11] Section I.1. Given a basis $\left\{e_{1}, e_{2}\right\}$ for the tangent plane $T\left(\mathbb{S}^{2}\right)$, the Christoffel symbols are defined by

$$
\nabla_{e_{i}} e_{j}=\Gamma_{i j}^{k} e_{k}+\Gamma_{i j}^{l} e_{l}, i, j, k, l=1,2 .
$$

For instance, considering as a basis the vectors $\left\{\frac{\partial}{\partial \theta}, \frac{\partial}{\partial \varphi}\right\}$ the Christoffel symbols can be shown to be equal to

$$
\Gamma_{\theta \varphi}^{\theta}=\Gamma_{\theta \theta}^{\theta}=\Gamma_{\varphi \varphi}^{\varphi}=\Gamma_{\theta \theta}^{\varphi}=0, \quad \Gamma_{\varphi \varphi}^{\theta}=-\sin \theta \cos \theta, \quad \Gamma_{\varphi \theta}^{\varphi}=\cot \theta .
$$

On the other hand, considering the orthonormal vectors $\frac{\partial}{\partial \theta}$ and $\frac{\partial}{\sin \theta \partial \varphi}$, using the linearity properties of the Levi Civita connection and the Leibnitz rule, we obtain also easily

$$
\nabla_{\frac{\partial}{\partial \theta}} \frac{\partial}{\partial \theta}=0, \quad \nabla_{\frac{\partial}{\partial \theta}} \frac{\partial}{\sin \theta \partial \varphi}=0, \quad \nabla_{\frac{\partial}{\sin \theta \partial \varphi}} \frac{\partial}{\partial \theta}=\cot \theta \frac{\partial}{\sin \theta \partial \varphi}, \quad \nabla_{\frac{\partial}{\sin \theta \partial \varphi}} \frac{\partial}{\sin \theta \partial \varphi}=-\cot \theta \frac{\partial}{\partial \theta} .
$$

Note indeed that in this framework the torsion-free property yields (taking $X=\frac{\partial}{\partial \theta}$ and $Y=\frac{\partial}{\sin \theta \partial \varphi}$ )

$$
X Y-Y X=\frac{\partial}{\partial \theta} \frac{\partial}{\sin \theta \partial \varphi}-\frac{\partial}{\sin \theta \partial \varphi} \frac{\partial}{\partial \theta}=-\cot \theta \frac{\partial}{\sin \theta \partial \varphi}=\nabla_{\frac{\partial}{\partial \theta}} \frac{\partial}{\sin \theta \partial \varphi}-\nabla_{\frac{\partial}{\sin \theta \partial \varphi}} \frac{\partial}{\partial \theta}=\nabla_{X} Y-\nabla_{Y} X
$$

The (covariant) Hessian is defined as the bilinear form $\left(\nabla^{2} f_{\ell}\right): T\left(\mathbb{S}^{2}\right) \times T\left(\mathbb{S}^{2}\right) \rightarrow \mathbb{R}$ such that

$$
\left(\nabla^{2} f_{\ell}\right)(X, Y):=X Y f_{\ell}-\nabla_{X} Y f_{\ell}
$$

in coordinates, the (covariant) Hessian matrix is hence obtained by replacing the elements of the basis $\partial_{1}, \partial_{2}$ in this expression, leading to

$$
\nabla^{2} f_{\ell}(x)=\left\{\left(\nabla^{2} f_{\ell}\right)\left(\partial_{a}, \partial_{a}\right)\right\}_{a, b=1,2}=\left\{\partial_{a} \partial_{b} f_{\ell}(x)-\nabla_{\partial_{a}} \partial_{b} f_{\ell}(x)\right\}_{a, b=1,2}
$$




$$
\begin{aligned}
& =\left(\begin{array}{cc}
\frac{\partial^{2} f_{\ell}(x)}{\partial \theta^{2}}-\Gamma_{\theta \theta}^{\theta} \frac{\partial f_{\ell}(x)}{\partial \theta}-\Gamma_{\theta \theta}^{\varphi} \frac{\partial f_{\ell}(x)}{\partial \varphi} & \frac{1}{\sin \theta_{x}}\left[\frac{\partial^{2} f_{\ell}(x)}{\partial \theta \partial \varphi}-\Gamma_{\varphi \theta}^{\varphi} \frac{\partial f_{\ell}(x)}{\partial \varphi}-\Gamma_{\theta \varphi}^{\theta} \frac{\partial f_{\ell}(x)}{\partial \theta}\right] \\
\frac{1}{\sin \theta_{x}}\left[\frac{\partial^{2} f_{\ell}(x)}{\partial \theta \partial \varphi}-\Gamma_{\varphi \theta}^{\varphi} \frac{\partial f_{\ell}(x)}{\partial \varphi}-\Gamma_{\theta \varphi}^{\theta} \frac{\partial f_{\ell}(x)}{\partial \theta}\right] & \frac{1}{\sin ^{2} \theta_{x}}\left[\frac{\partial^{2} f_{\ell}(x)}{\partial \varphi^{2}}-\Gamma_{\varphi \varphi}^{\varphi} \frac{\partial f_{\ell}(x)}{\partial \varphi}-\Gamma_{\varphi \varphi}^{\theta} \frac{\partial f_{\ell}(x)}{\partial \theta}\right]
\end{array}\right) \\
& =\left(\begin{array}{cc}
\frac{\partial^{2} f_{\ell}(x)}{\partial \theta^{2}} & \frac{1}{\sin \theta}\left(\frac{\partial^{2} f_{\ell}(x)}{\partial \theta \partial \varphi}-\cot \theta \frac{\partial f_{\ell}(x)}{\partial \varphi}\right) \\
\frac{1}{\sin \theta}\left(\frac{\partial^{2} f_{\ell}(x)}{\partial \theta \partial \varphi}-\cot \theta \frac{\partial f_{\ell}(x)}{\partial \varphi}\right) & \frac{1}{\sin ^{2} \theta}\left(\frac{\partial^{2} f_{\ell}(x)}{\partial \varphi^{2}}+\sin \theta \cos \theta \frac{\partial f_{\ell}(x)}{\partial \theta}\right)
\end{array}\right) .
\end{aligned}
$$

It is important to note that, as expected

$$
\operatorname{Tr}\left(\nabla^{2} f_{\ell}(x)\right)=\frac{\partial^{2} f_{\ell}(x)}{\partial \theta^{2}}+\frac{1}{\sin ^{2} \theta}\left(\frac{\partial^{2} f_{\ell}(x)}{\partial \varphi^{2}}+\sin \theta \cos \theta \frac{\partial f_{\ell}(x)}{\partial \theta}\right)=\Delta_{S^{2}} f_{\ell}(x),
$$

i.e., the trace of the Hessian operator corresponds to the Laplacian.

\section{Appendix B: Technical Lemmas}

This section collects the analytic expressions for the derivatives that we have exploited to prove our results.

\subsection{Covariances of first derivatives.}

Lemma 11. For all points $x=\left(\theta_{x}, \varphi_{x}\right) \in \mathbb{S}^{2} \backslash\{N, S\}$,

$$
\begin{aligned}
& \left.\mathbb{E}\left[\partial_{1 ; x} f_{\ell}(x) \partial_{1 ; y} f_{\ell}(y)\right]\right|_{x=y}=P_{\ell}^{\prime}(1), \\
& \left.\mathbb{E}\left[\partial_{1 ; x} f_{\ell}(x) \partial_{2 ; y} f_{\ell}(y)\right]\right|_{x=y}=0, \\
& \left.\mathbb{E}\left[\partial_{2 ; x} f_{\ell}(x) \partial_{2 ; y} f_{\ell}(y)\right]\right|_{x=y}=P_{\ell}^{\prime}(1) .
\end{aligned}
$$

Proof. We have that

$$
\begin{aligned}
& \left.\mathbb{E}\left[\partial_{1 ; x} f_{\ell}(x) \partial_{1 ; y} f_{\ell}(y)\right]\right|_{x=y} \\
& =\left.P_{\ell}^{\prime \prime}(\langle x, y\rangle)\left\{-\cos \theta_{x} \sin \theta_{y}+\sin \theta_{x} \cos \theta_{y} \cos \left(\varphi_{x}-\varphi_{y}\right)\right\}\left\{-\sin \theta_{x} \cos \theta_{y}+\cos \theta_{x} \sin \theta_{y} \cos \left(\varphi_{x}-\varphi_{y}\right)\right\}\right|_{x=y} \\
& +\left.P_{\ell}^{\prime}(\langle x, y\rangle)\left\{\sin \theta_{x} \sin \theta_{y}+\cos \theta_{x} \cos \theta_{y} \cos \left(\varphi_{x}-\varphi_{y}\right)\right\}\right|_{x=y} \\
& =\left.P_{\ell}^{\prime \prime}(1)\left\{-\cos \theta_{x} \sin \theta_{x}+\sin \theta_{x} \cos \theta_{x}\right\}\left\{-\sin \theta_{x} \cos \theta_{x}+\cos \theta_{x} \sin \theta_{x}\right\}\right|_{x=y} \\
& +\left.P_{\ell}^{\prime}(1)\left\{\sin ^{2} \theta_{x}+\cos ^{2} \theta_{x}\right\}\right|_{x=y}=P_{\ell}^{\prime}(1),
\end{aligned}
$$

moreover

$$
\begin{aligned}
& \left.\mathbb{E}\left[\partial_{1 ; x} f_{\ell}(x) \partial_{2 ; y} f_{\ell}(y)\right]\right|_{x=y} \\
& =\left.\frac{1}{\sin \theta_{y}} P_{\ell}^{\prime \prime}(\langle x, y\rangle)\left\{-\sin \theta_{x} \cos \theta_{y}+\cos \theta_{x} \sin \theta_{y} \cos \left(\varphi_{x}-\varphi_{y}\right)\right\}\left\{\sin \theta_{x} \sin \theta_{y} \sin \left(\varphi_{x}-\varphi_{y}\right)\right\}\right|_{x=y} \\
& +\left.\frac{1}{\sin \theta_{y}} P_{\ell}^{\prime}(\langle x, y\rangle) \cos \theta_{x} \sin \theta_{y} \sin \left(\varphi_{x}-\varphi_{y}\right)\right|_{x=y} \\
& =\left.\frac{1}{\sin \theta_{x}} P_{\ell}^{\prime \prime}(1)\left\{-\sin \theta_{x} \cos \theta_{x}+\cos \theta_{x} \sin \theta_{x}\right\} \sin ^{2} \theta_{x} \sin \left(\varphi_{x}-\varphi_{x}\right)\right|_{x=y} \\
& +\left.\frac{1}{\sin \theta_{x}} P_{\ell}^{\prime}(\langle x, y\rangle) \cos \theta_{x} \sin \theta_{x} \sin \left(\varphi_{x}-\varphi_{x}\right)\right|_{x=y}=0,
\end{aligned}
$$

and finally

$$
\begin{aligned}
& \left.\mathbb{E}\left[\partial_{2 ; x} f_{\ell}(x) \partial_{2 ; y} f_{\ell}(y)\right]\right|_{x=y} \\
& =-\frac{1}{\sin \theta_{y}} \frac{1}{\sin \theta_{x}} P_{\ell}^{\prime \prime}(\langle x, y\rangle)\left\{\sin \theta_{x} \sin \theta_{y} \sin ^{2}\left(\varphi_{x}-\varphi_{y}\right)\right\}+\left.P_{\ell}^{\prime}(\langle x, y\rangle) \cos \left(\varphi_{x}-\varphi_{y}\right)\right|_{x=y} \\
& =-P_{\ell}^{\prime \prime}(1) \sin ^{2}\left(\varphi_{x}-\varphi_{x}\right)+\left.P_{\ell}^{\prime}(1) \cos \left(\varphi_{x}-\varphi_{x}\right)\right|_{x=y}=P_{\ell}^{\prime}(1) .
\end{aligned}
$$




\subsection{Cross-covariances of first- and second-order derivatives.}

Lemma 12. For all points $x=\left(\theta_{x}, \varphi_{x}\right) \in \mathbb{S}^{2} \backslash\{N, S\}$,

$$
\left.\mathbb{E}\left[\partial_{11 ; x} f_{\ell}(x) \partial_{1 ; y} f_{\ell}(y)\right]\right|_{x=y}=\left.\mathbb{E}\left[\partial_{11 ; x} f_{\ell}(x) \partial_{2 ; y} f_{\ell}(y)\right]\right|_{x=y}=0 .
$$

Proof. We have that

$$
\begin{aligned}
& \left.\mathbb{E}\left[\partial_{11 ; x} f_{\ell}(x) \partial_{1 ; y} f_{\ell}(y)\right]\right|_{x=y} \\
& =\left.\partial_{11 ; x}\left\{P_{\ell}^{\prime}(\langle x, y\rangle)\left\{-\cos \theta_{x} \sin \theta_{y}+\sin \theta_{x} \cos \theta_{y} \cos \left(\varphi_{x}-\varphi_{y}\right)\right\}\right\}\right|_{x=y} \\
& =\left.\partial_{1 ; x}\left\{P_{\ell}^{\prime \prime}(\langle x, y\rangle)\left\{-\cos \theta_{x} \sin \theta_{y}+\sin \theta_{x} \cos \theta_{y} \cos \left(\varphi_{x}-\varphi_{y}\right)\right\}^{2}\right\}\right|_{x=y} \\
& +\left.\partial_{1 ; x}\left\{P_{\ell}^{\prime}(\langle x, y\rangle)\left\{\cos \theta_{x} \sin \theta_{y}-\sin \theta_{x} \cos \theta_{y} \cos \left(\varphi_{x}-\varphi_{y}\right)\right\}\right\}\right|_{x=y} \\
& =\left.\left\{P_{\ell}^{\prime \prime \prime}(\langle x, y\rangle)\left\{-\cos \theta_{x} \sin \theta_{y}+\sin \theta_{x} \cos \theta_{y} \cos \left(\varphi_{x}-\varphi_{y}\right)\right\}^{3}\right\}\right|_{x=y} \\
& +\left.2\left\{P_{\ell}^{\prime \prime}(\langle x, y\rangle)\left\{-\cos \theta_{x} \sin \theta_{y}+\sin \theta_{x} \cos \theta_{y} \cos \left(\varphi_{x}-\varphi_{y}\right)\right\}\left\{\cos \theta_{x} \sin \theta_{y}-\sin \theta_{x} \cos \theta_{y} \cos \left(\varphi_{x}-\varphi_{y}\right)\right\}\right\}\right|_{x=y} \\
& +\left.\left\{P_{\ell}^{\prime \prime}(\langle x, y\rangle)\left\{\cos \theta_{x} \sin \theta_{y}-\sin \theta_{x} \cos \theta_{y} \cos \left(\varphi_{x}-\varphi_{y}\right)\right\}\right\}\right|_{x=y} \\
& +\left.\left\{P_{\ell}^{\prime}(\langle x, y\rangle)\left\{-\cos \theta_{x} \sin \theta_{y}+\sin \theta_{x} \cos \theta_{y} \cos \left(\varphi_{x}-\varphi_{y}\right)\right\}\right\}\right|_{x=y}=0
\end{aligned}
$$

Likewise

$$
\begin{aligned}
& \left.\mathbb{E}\left[\partial_{11 ; x} f_{\ell}(x) \partial_{2 ; y} f_{\ell}(y)\right]\right|_{x=y} \\
& =\left.\frac{1}{\sin \theta_{y}}\left\{P_{\ell}^{\prime \prime \prime}(\langle x, y\rangle)\left\{-\cos \theta_{x} \sin \theta_{y}+\sin \theta_{x} \sin \theta_{y} \cos \left(\varphi_{x}-\varphi_{y}\right)\right\}^{2}\left\{\sin \theta_{x} \sin \theta_{y} \sin \left(\varphi_{x}-\varphi_{y}\right)\right\}\right\}\right|_{x=y} \\
& +\left.\frac{1}{\sin \theta_{y}}\left\{P_{\ell}^{\prime \prime}(\langle x, y\rangle)\left\{\sin \theta_{x} \sin \theta_{y}+\cos \theta_{x} \sin \theta_{y} \cos \left(\varphi_{x}-\varphi_{y}\right)\right\}\left\{\sin \theta_{x} \sin \theta_{y} \sin \left(\varphi_{x}-\varphi_{y}\right)\right\}\right\}\right|_{x=y} \\
& +\left.\frac{1}{\sin \theta_{y}}\left\{P_{\ell}^{\prime \prime}(\langle x, y\rangle)\left\{-\cos \theta_{x} \sin \theta_{y}+\sin \theta_{x} \sin \theta_{y} \cos \left(\varphi_{x}-\varphi_{y}\right)\right\}\left\{\cos \theta_{x} \sin \theta_{y} \sin \left(\varphi_{x}-\varphi_{y}\right)\right\}\right\}\right|_{x=y} \\
& +\left.\frac{1}{\sin \theta_{y}}\left\{P_{\ell}^{\prime \prime}(\langle x, y\rangle)\left\{\cos \theta_{x} \sin \theta_{y} \sin \left(\varphi_{x}-\varphi_{y}\right)\right\}\left\{-\cos \theta_{x} \sin \theta_{y}+\sin \theta_{x} \sin \theta_{y} \cos \left(\varphi_{x}-\varphi_{y}\right)\right\}\right\}\right|_{x=y} \\
& -\left.\frac{1}{\sin \theta_{y}}\left\{P_{\ell}^{\prime}(\langle x, y\rangle)\left\{\sin \theta_{x} \sin \theta_{y} \sin \left(\varphi_{x}-\varphi_{y}\right)\right\}\right\}\right|_{x=y}=0 .
\end{aligned}
$$

Lemma 13. For all points $x=\left(\theta_{x}, \varphi_{x}\right) \in \mathbb{S}^{2} \backslash\{N, S\}$,

$$
\begin{aligned}
& \left.\mathbb{E}\left[\partial_{22 ; x} f_{\ell}(x) \partial_{1 ; y} f_{\ell}(y)\right]\right|_{x=y}=-\cot \theta_{x} P_{\ell}^{\prime}(1), \\
& \left.\mathbb{E}\left[\partial_{22 ; x} f_{\ell}(x) \partial_{2 ; y} f_{\ell}(y)\right]\right|_{x=y}=0 .
\end{aligned}
$$

Proof. We have that

$$
\begin{aligned}
& \left.\mathbb{E}\left[\partial_{22 ; x} f_{\ell}(x) \partial_{1 ; y} f_{\ell}(y)\right]\right|_{x=y} \\
& =\left.\partial_{22 ; x}\left\{P_{\ell}^{\prime}(\langle x, y\rangle)\left\{-\cos \theta_{x} \sin \theta_{y}+\cos \theta_{x} \sin \theta_{y} \cos \left(\varphi_{x}-\varphi_{y}\right)\right\}\right\}\right|_{x=y} \\
& =\left.\frac{1}{\sin ^{2} \theta_{x}}\left\{P_{\ell}^{\prime \prime \prime}(\langle x, y\rangle)\left\{-\sin \theta_{x} \sin \theta_{y} \sin \left(\varphi_{x}-\varphi_{y}\right)\right\}\left\{-\cos \theta_{x} \sin \theta_{y}+\cos \theta_{x} \sin \theta_{y} \cos \left(\varphi_{x}-\varphi_{y}\right)\right\}^{2}\right\}\right|_{x=y} \\
& +\left.\frac{1}{\sin ^{2} \theta_{x}}\left\{P_{\ell}^{\prime \prime}(\langle x, y\rangle)\left\{-\sin \theta_{x} \sin \theta_{y} \cos \left(\varphi_{x}-\varphi_{y}\right)\right\}\left\{-\cos \theta_{x} \sin \theta_{y}+\cos \theta_{x} \sin \theta_{y} \cos \left(\varphi_{x}-\varphi_{y}\right)\right\}\right\}\right|_{x=y} \\
& +\left.\frac{1}{\sin ^{2} \theta_{x}}\left\{P_{\ell}^{\prime \prime}(\langle x, y\rangle)\left\{-\sin \theta_{x} \sin \theta_{y} \sin \left(\varphi_{x}-\varphi_{y}\right)\right\}\left\{-\cos \theta_{x} \sin \theta_{y} \sin \left(\varphi_{x}-\varphi_{y}\right)\right\}\right\}\right|_{x=y} \\
& +\left.\frac{1}{\sin ^{2} \theta_{x}}\left\{P_{\ell}^{\prime \prime}(\langle x, y\rangle)\left\{-\sin \theta_{x} \sin \theta_{y} \sin \left(\varphi_{x}-\varphi_{y}\right)\right\}\left\{-\cos \theta_{x} \sin \theta_{y} \sin \left(\varphi_{x}-\varphi_{y}\right)\right\}\right\}\right|_{x=y} \\
& +\left.\frac{1}{\sin ^{2} \theta_{x}}\left\{P_{\ell}^{\prime}(\langle x, y\rangle)\left\{-\cos \theta_{x} \sin \theta_{y} \cos \left(\varphi_{x}-\varphi_{y}\right)\right\}\right\}\right|_{x=y}=-\cot \theta_{x} P_{\ell}^{\prime}(1)
\end{aligned}
$$


The proof of the second result is similar:

$$
\begin{aligned}
& \left.\mathbb{E}\left[\partial_{22 ; x} f_{\ell}(x) \partial_{2 ; y} f_{\ell}(y)\right]\right|_{x=y} \\
& =\left.\frac{1}{\sin \theta_{y}} \partial_{22 ; x}\left\{P_{\ell}^{\prime}(\langle x, y\rangle)\left\{\sin \theta_{x} \sin \theta_{y} \sin \left(\varphi_{x}-\varphi_{y}\right)\right\}\right\}\right|_{x=y} \\
& =\left.\frac{1}{\sin ^{2} \theta_{x}} \frac{1}{\sin \theta_{y}}\left\{P_{\ell}^{\prime \prime \prime}(\langle x, y\rangle)\left\{\sin \theta_{x} \sin \theta_{y} \sin \left(\varphi_{x}-\varphi_{y}\right)\right\}^{3}\right\}\right|_{x=y} \\
& +\left.\frac{1}{\sin ^{2} \theta_{x}} \frac{1}{\sin \theta_{y}} 2\left\{P_{\ell}^{\prime \prime}(\langle x, y\rangle) 2\left\{\sin \theta_{x} \sin \theta_{y} \sin \left(\varphi_{x}-\varphi_{y}\right)\right\}\left\{\sin \theta_{x} \sin \theta_{y} \cos \left(\varphi_{x}-\varphi_{y}\right)\right\}\right\}\right|_{x=y} \\
& -\left.\frac{1}{\sin ^{2} \theta_{x}} \frac{1}{\sin \theta_{y}}\left\{P_{\ell}^{\prime \prime}(\langle x, y\rangle)\left\{\sin \theta_{x} \sin \theta_{y} \sin \left(\varphi_{x}-\varphi_{y}\right)\right\}\left\{\sin \theta_{x} \sin \theta_{y} \cos \left(\varphi_{x}-\varphi_{y}\right)\right\}\right\}\right|_{x=y} \\
& -\left.\frac{1}{\sin ^{2} \theta_{x}} \frac{1}{\sin \theta_{y}}\left\{P_{\ell}^{\prime}(\langle x, y\rangle)\left\{\sin \theta_{x} \sin \theta_{y} \sin \left(\varphi_{x}-\varphi_{y}\right)\right\}\right\}\right|_{x=y}=0
\end{aligned}
$$

For the cross-derivatives, we shall need also:

Lemma 14. For all points $x=\left(\theta_{x}, \varphi_{x}\right) \in \mathbb{S}^{2} \backslash\{N, S\}$,

$$
\begin{aligned}
& \left.\mathbb{E}\left[\partial_{21 ; x} f_{\ell}(x) \partial_{1 ; y} f_{\ell}(y)\right]\right|_{x=y}=0, \\
& \left.\mathbb{E}\left[\partial_{21 ; x} f_{\ell}(x) \partial_{2 ; y} f_{\ell}(y)\right]\right|_{x=y}=\cot \theta_{x} P_{\ell}^{\prime}(1) .
\end{aligned}
$$

Proof. We have that

$$
\begin{aligned}
& \left.\mathbb{E}\left[\partial_{12 ; x} f_{\ell}(x) \partial_{1 ; y} f_{\ell}(y)\right]\right|_{x=y} \\
& =-\left.\frac{1}{\sin \theta_{x}}\left\{P_{\ell}^{\prime \prime \prime}(\langle x, y\rangle)\left\{\sin \theta_{x} \sin \theta_{y} \sin \left(\varphi_{x}-\varphi_{y}\right)\right\}\left\{-\cos \theta_{x} \sin \theta_{y}+\sin \theta_{x} \cos \theta_{y} \cos \left(\varphi_{x}-\varphi_{y}\right)\right\}^{2}\right\}\right|_{x=y} \\
& -\left.\frac{1}{\sin \theta_{x}}\left\{P_{\ell}^{\prime \prime}(\langle x, y\rangle)\left\{\cos \theta_{x} \sin \theta_{y} \sin \left(\varphi_{x}-\varphi_{y}\right)\right\}\left\{-\cos \theta_{x} \sin \theta_{y}+\sin \theta_{x} \cos \theta_{y} \cos \left(\varphi_{x}-\varphi_{y}\right)\right\}\right\}\right|_{x=y} \\
& -\left.\frac{1}{\sin \theta_{x}}\left\{P_{\ell}^{\prime \prime}(\langle x, y\rangle)\left\{\sin \theta_{x} \sin \theta_{y} \sin \left(\varphi_{x}-\varphi_{y}\right)\right\}\left\{\sin \theta_{x} \sin \theta_{y}+\cos \theta_{x} \cos \theta_{y} \cos \left(\varphi_{x}-\varphi_{y}\right)\right\}\right\}\right|_{x=y} \\
& -\left.\frac{1}{\sin \theta_{x}}\left\{P_{\ell}^{\prime \prime}(\langle x, y\rangle)\left\{-\sin \theta_{x} \cos \theta_{y}+\cos \theta_{x} \sin \theta_{y} \cos \left(\varphi_{x}-\varphi_{y}\right)\right\}\left\{\sin \theta_{x} \cos \theta_{y} \sin \left(\varphi_{x}-\varphi_{y}\right)\right\}\right\}\right|_{x=y} \\
& -\left.\frac{1}{\sin \theta_{x}}\left\{P_{\ell}^{\prime}(\langle x, y\rangle)\left\{\cos \theta_{x} \cos \theta_{y} \sin \left(\varphi_{x}-\varphi_{y}\right)\right\}\right\}\right|_{x=y}=0
\end{aligned}
$$

and more importantly

$$
\begin{aligned}
& \left.\mathbb{E}\left[\partial_{12 ; x} f_{\ell}(x) \partial_{2 ; y} f_{\ell}(y)\right]\right|_{x=y} \\
& =\left.\frac{1}{\sin \theta_{y}} \partial_{12 ; x}\left\{P_{\ell}^{\prime}(\langle x, y\rangle)\left\{\sin \theta_{x} \sin \theta_{y} \sin \left(\varphi_{x}-\varphi_{y}\right)\right\}\right\}\right|_{x=y} \\
& =-\left.\frac{1}{\sin \theta_{x}} \frac{1}{\sin \theta_{y}} \partial_{1 ; x}\left\{P_{\ell}^{\prime \prime}(\langle x, y\rangle)\left\{\sin \theta_{x} \sin \theta_{y} \sin \left(\varphi_{x}-\varphi_{y}\right)\right\}^{2}\right\}\right|_{x=y} \\
& +\left.\frac{1}{\sin \theta_{x}} \frac{1}{\sin \theta_{y}} \partial_{1 ; x}\left\{P_{\ell}^{\prime}(\langle x, y\rangle)\left\{\sin \theta_{x} \sin \theta_{y} \cos \left(\varphi_{x}-\varphi_{y}\right)\right\}\right\}\right|_{x=y} \\
& =-\left.\frac{1}{\sin \theta_{x}} \frac{1}{\sin \theta_{y}}\left\{P_{\ell}^{\prime \prime \prime}(\langle x, y\rangle)\left\{-\sin \theta_{x} \cos \theta_{y}+\sin \theta_{x} \sin \theta_{y} \cos \left(\varphi_{x}-\varphi_{y}\right)\right\}\left\{\sin \theta_{x} \sin \theta_{y} \sin \left(\varphi_{x}-\varphi_{y}\right)\right\}^{2}\right\}\right|_{x=y} \\
& -\left.\frac{1}{\sin \theta_{x}} \frac{1}{\sin \theta_{y}}\left\{P_{\ell}^{\prime \prime}(\langle x, y\rangle) 2\left\{\sin \theta_{x} \sin \theta_{y} \sin \left(\varphi_{x}-\varphi_{y}\right)\right\}\left\{\cos \theta_{x} \sin \theta_{y} \sin \left(\varphi_{x}-\varphi_{y}\right)\right\}\right\}\right|_{x=y} \\
& +\left.\frac{1}{\sin \theta_{x}} \frac{1}{\sin \theta_{y}}\left\{P_{\ell}^{\prime \prime}(\langle x, y\rangle)\left\{-\sin \theta_{x} \cos \theta_{y}+\cos \theta_{x} \sin \theta_{y} \cos \left(\varphi_{x}-\varphi_{y}\right)\right\}\left\{\sin \theta_{x} \sin \theta_{y} \cos \left(\varphi_{x}-\varphi_{y}\right)\right\}\right\}\right|_{x=y} \\
& +\left.\frac{1}{\sin \theta_{x}} \frac{1}{\sin \theta_{y}}\left\{P_{\ell}^{\prime}(\langle x, y\rangle)\left\{\cos \theta_{x} \sin \theta_{y} \cos \left(\varphi_{x}-\varphi_{y}\right)\right\}\right\}\right|_{x=y}=\cot \theta_{x} P_{\ell}^{\prime}(1)
\end{aligned}
$$


7.3. Covariances of second-order derivatives. Let us now consider the second-order derivatives.

Lemma 15. For all points $x=\left(\theta_{x}, \varphi_{x}\right) \in \mathbb{S}^{2} \backslash\{N, S\}$,

$$
\left.\mathbb{E}\left[\partial_{11 ; x} f_{\ell}(x) \partial_{11 ; y} f_{\ell}(y)\right]\right|_{x=y}=3 P_{\ell}^{\prime \prime}(1)+P_{\ell}^{\prime}(1) .
$$

Proof. By an explicit computation of derivatives, we have that

$$
\left.\mathbb{E}\left[\partial_{11 ; x} f_{\ell}(x) \partial_{11 ; y} f_{\ell}(y)\right]\right|_{x=y}=A+B+C+D,
$$

where

$$
\begin{aligned}
A & =\left.P_{\ell}^{\prime \prime \prime \prime}(\langle x, y\rangle)\left\{-\sin \theta_{x} \cos \theta_{y}+\cos \theta_{x} \sin \theta_{y} \cos \left(\varphi_{x}-\varphi_{x}\right)\right\}^{2}\left\{-\cos \theta_{x} \sin \theta_{y}+\sin \theta_{x} \cos \theta_{y} \cos \left(\varphi_{x}-\varphi_{x}\right)\right\}^{2}\right|_{x=y} \\
& +\left.P_{\ell}^{\prime \prime \prime}(\langle x, y\rangle)\left\{-\cos \theta_{x} \cos \theta_{y}-\sin \theta_{x} \sin \theta_{y} \cos \left(\varphi_{x}-\varphi_{x}\right)\right\}\left\{-\cos \theta_{x} \sin \theta_{y}+\sin \theta_{x} \cos \theta_{y} \cos \left(\varphi_{x}-\varphi_{x}\right)\right\}^{2}\right|_{x=y} \\
& +P_{\ell}^{\prime \prime \prime}(\langle x, y\rangle)\left\{-\sin \theta_{x} \cos \theta_{y}+\cos \theta_{x} \sin \theta_{y} \cos \left(\varphi_{x}-\varphi_{x}\right)\right\} \\
& \times\left. 2\left\{\sin \theta_{x} \sin \theta_{y}+\cos \theta_{x} \cos \theta_{y} \cos \left(\varphi_{x}-\varphi_{x}\right)\right\}\left\{-\cos \theta_{x} \sin \theta_{y}+\sin \theta_{x} \cos \theta_{y} \cos \left(\varphi_{x}-\varphi_{x}\right)\right\}\right|_{x=y} \\
= & 0 \\
B & =\left.P_{\ell}^{\prime \prime \prime}(\langle x, y\rangle) 2\left\{-\cos \theta_{x} \sin \theta_{y}+\sin \theta_{x} \cos \theta_{y} \cos \left(\varphi_{x}-\varphi_{x}\right)\right\}^{2}\left\{\sin \theta_{x} \sin \theta_{y}+\cos \theta_{x} \cos \theta_{y} \cos \left(\varphi_{x}-\varphi_{x}\right)\right\}\right|_{x=y} \\
& +\left.P_{\ell}^{\prime \prime}(\langle x, y\rangle) 2\left\{\sin \theta_{x} \sin \theta_{y}+\cos \theta_{x} \cos \theta_{y} \cos \left(\varphi_{x}-\varphi_{x}\right)\right\}\left\{\sin \theta_{x} \sin \theta_{y}+\cos \theta_{x} \cos \theta_{y} \cos \left(\varphi_{x}-\varphi_{x}\right)\right\}\right|_{x=y} \\
& +\left.P_{\ell}^{\prime \prime}(\langle x, y\rangle) 2\left\{-\cos \theta_{x} \sin \theta_{y}+\sin \theta_{x} \cos \theta_{y} \cos \left(\varphi_{x}-\varphi_{x}\right)\right\}\left\{\cos \theta_{x} \sin \theta_{y}-\sin \theta_{x} \cos \theta_{y} \cos \left(\varphi_{x}-\varphi_{x}\right)\right\}\right|_{x=y} \\
& =2 P_{\ell}^{\prime \prime}(1), \\
C & =\left.P_{\ell}^{\prime \prime \prime}(\langle x, y\rangle)\left\{-\sin \theta_{x} \cos \theta_{y}+\cos \theta_{x} \sin \theta_{y} \cos \left(\varphi_{x}-\varphi_{x}\right)\right\}^{2}\left\{-\cos \theta_{x} \cos \theta_{y}-\sin \theta_{x} \sin \theta_{y} \cos \left(\varphi_{x}-\varphi_{x}\right)\right\}\right|_{x=y} \\
& +\left.P_{\ell}^{\prime \prime}(\langle x, y\rangle)\left\{-\cos \theta_{x} \cos \theta_{y}-\sin \theta_{x} \sin \theta_{y} \cos \left(\varphi_{x}-\varphi_{x}\right)\right\}\left\{-\cos \theta_{x} \cos \theta_{y}-\sin \theta_{x} \sin \theta_{y} \cos \left(\varphi_{x}-\varphi_{x}\right)\right\}\right|_{x=y} \\
& +\left.P_{\ell}^{\prime \prime}(\langle x, y\rangle)\left\{-\sin \theta_{x} \cos \theta_{y}+\cos \theta_{x} \sin \theta_{y} \cos \left(\varphi_{x}-\varphi_{x}\right)\right\}\left\{\sin \theta_{x} \cos \theta_{y}-\cos \theta_{x} \sin \theta_{y} \cos \left(\varphi_{x}-\varphi_{x}\right)\right\}\right|_{x=y} \\
& =P_{\ell}^{\prime \prime}(1)
\end{aligned}
$$

and finally

$$
\begin{aligned}
D & =\left.P_{\ell}^{\prime \prime}(\langle x, y\rangle)\left\{-\sin \theta_{x} \cos \theta_{y}+\cos \theta_{x} \sin \theta_{y} \cos \left(\varphi_{x}-\varphi_{x}\right)\right\}\left\{\sin \theta_{x} \cos \theta_{y}-\cos \theta_{x} \sin \theta_{y} \cos \left(\varphi_{x}-\varphi_{x}\right)\right\}\right|_{x=y} \\
& +\left.P_{\ell}^{\prime}(\langle x, y\rangle)\left\{\cos \theta_{x} \cos \theta_{y}+\sin \theta_{x} \sin \theta_{y} \cos \left(\varphi_{x}-\varphi_{x}\right)\right\}\right|_{x=y} \\
& =P_{\ell}^{\prime}(1) .
\end{aligned}
$$

Our next result is the following:

Lemma 16. For all points $x=\left(\theta_{x}, \varphi_{x}\right) \in \mathbb{S}^{2} \backslash\{N, S\}$,

$$
\left.\mathbb{E}\left[\partial_{22 ; x} f_{\ell}(x) \partial_{11 ; y} f_{\ell}(y)\right]\right|_{x=y}=P_{\ell}^{\prime \prime}(1)+P_{\ell}^{\prime}(1) .
$$

Proof. Again, evaluation of derivatives gives

$$
\begin{aligned}
& \left.\mathbb{E}\left[\partial_{22 ; x} f_{\ell}(x) \partial_{11 ; y} f_{\ell}(y)\right]\right|_{x=y} \\
& =\left.\partial_{2 ; x} P_{\ell}^{\prime \prime \prime}(\langle x, y\rangle)\left\{-\sin \theta_{y} \sin \left(\varphi_{x}-\varphi_{x}\right)\right\}\left\{-\cos \theta_{x} \sin \theta_{y}+\sin \theta_{x} \cos \theta_{y} \cos \left(\varphi_{x}-\varphi_{y}\right)\right\}^{2}\right|_{x=y} \\
& +\left.\partial_{2 ; x} P_{\ell}^{\prime \prime}(\langle x, y\rangle) 2\left\{-\cos \theta_{x} \sin \theta_{y}+\sin \theta_{x} \cos \theta_{y} \cos \left(\varphi_{x}-\varphi_{y}\right)\right\}\left\{-\cos \theta_{y} \sin \left(\varphi_{x}-\varphi_{y}\right)\right\}\right|_{y} \\
& +\left.\partial_{2 ; x} P_{\ell}^{\prime \prime}(\langle x, y\rangle)\left\{-\sin \theta_{y} \sin \left(\varphi_{x}-\varphi_{y}\right)\right\}\left\{-\cos \theta_{x} \cos \theta_{y}-\sin \theta_{x} \sin \theta_{y} \cos \left(\varphi_{x}-\varphi_{y}\right)\right\}\right|_{x=y} \\
& +\left.\partial_{2 ; x} P_{\ell}^{\prime}(\langle x, y\rangle)\left\{\sin \theta_{y} \sin \left(\varphi_{x}-\varphi_{y}\right)\right\}\right|_{x=y} \\
& =A+B+C+D,
\end{aligned}
$$

where

$$
\begin{aligned}
A= & \frac{1}{\sin \theta_{x}} P_{\ell}^{\prime \prime \prime \prime}(\langle x, y\rangle)\left\{-\sin \theta_{y} \sin \theta_{x} \sin \left(\varphi_{x}-\varphi_{x}\right)\right\} \\
& \times\left.\left\{-\sin \theta_{y} \sin \left(\varphi_{x}-\varphi_{x}\right)\right\}\left\{-\cos \theta_{x} \sin \theta_{y}+\sin \theta_{x} \cos \theta_{y} \cos \left(\varphi_{x}-\varphi_{y}\right)\right\}^{2}\right|_{x=y}
\end{aligned}
$$




$$
\begin{aligned}
& +\left.\frac{1}{\sin \theta_{x}} P_{\ell}^{\prime \prime \prime}(\langle x, y\rangle)\left\{-\sin \theta_{y} \cos \left(\varphi_{x}-\varphi_{x}\right)\right\}\left\{-\cos \theta_{x} \sin \theta_{y}+\sin \theta_{x} \cos \theta_{y} \cos \left(\varphi_{x}-\varphi_{y}\right)\right\}^{2}\right|_{x=y} \\
& +\left.\frac{1}{\sin \theta_{x}} P_{\ell}^{\prime \prime \prime}(\langle x, y\rangle)\left\{-\sin \theta_{y} \cos \left(\varphi_{x}-\varphi_{x}\right)\right\}\left\{-\cos \theta_{x} \sin \theta_{y}+\sin \theta_{x} \cos \theta_{y} \cos \left(\varphi_{x}-\varphi_{y}\right)\right\}^{2}\right|_{x=y} \\
& =0,
\end{aligned}
$$

$$
\begin{aligned}
B & =\left.P_{\ell}^{\prime \prime \prime}(\langle x, y\rangle)\left\{-\sin \theta_{y} \sin \left(\varphi_{x}-\varphi_{y}\right)\right\} 2\left\{-\cos \theta_{x} \sin \theta_{y}+\sin \theta_{x} \cos \theta_{y} \cos \left(\varphi_{x}-\varphi_{y}\right)\right\}\left\{-\cos \theta_{y} \sin \left(\varphi_{x}-\varphi_{y}\right)\right\}\right|_{x=y} \\
& +\left.P_{\ell}^{\prime \prime}(\langle x, y\rangle) 2\left\{-\cos \theta_{y} \sin \left(\varphi_{x}-\varphi_{y}\right)\right\}\left\{-\cos \theta_{y} \sin \left(\varphi_{x}-\varphi_{y}\right)\right\}\right|_{x=y} \\
& +\left.\frac{1}{\sin \theta_{x}} P_{\ell}^{\prime \prime}(\langle x, y\rangle) 2\left\{-\cos \theta_{x} \sin \theta_{y}+\sin \theta_{x} \cos \theta_{y} \cos \left(\varphi_{x}-\varphi_{y}\right)\right\}\left\{-\cos \theta_{y} \cos \left(\varphi_{x}-\varphi_{y}\right)\right\}\right|_{x=y} \\
& =0,
\end{aligned}
$$

$$
\begin{aligned}
C & =\left.P_{\ell}^{\prime \prime \prime}(\langle x, y\rangle)\left\{-\sin \theta_{y} \sin \left(\varphi_{x}-\varphi_{y}\right)\right\}^{2}\left\{-\cos \theta_{x} \cos \theta_{y}-\sin \theta_{x} \sin \theta_{y} \cos \left(\varphi_{x}-\varphi_{y}\right)\right\}\right|_{x=y} \\
& +\left.\frac{1}{\sin \theta_{x}} P_{\ell}^{\prime \prime}(\langle x, y\rangle)\left\{-\sin \theta_{y} \cos \left(\varphi_{x}-\varphi_{y}\right)\right\}\left\{-\cos \theta_{x} \cos \theta_{y}-\sin \theta_{x} \sin \theta_{y} \cos \left(\varphi_{x}-\varphi_{y}\right)\right\}\right|_{x=y} \\
& +\left.\partial_{2 ; x} P_{\ell}^{\prime \prime}(\langle x, y\rangle)\left\{-\sin \theta_{y} \sin \left(\varphi_{x}-\varphi_{y}\right)\right\}\left\{\sin \theta_{y} \sin \left(\varphi_{x}-\varphi_{y}\right)\right\}\right|_{x=y} \\
& =P_{\ell}^{\prime \prime}(1)
\end{aligned}
$$

and finally

$$
\begin{aligned}
D & =\left.\frac{1}{\sin \theta_{x}} P_{\ell}^{\prime \prime}(\langle x, y\rangle)\left\{-\sin \theta_{x} \sin \theta_{y} \sin \left(\varphi_{x}-\varphi_{y}\right)\right\}\left\{\sin \theta_{y} \sin \left(\varphi_{x}-\varphi_{y}\right)\right\}\right|_{x=y} \\
& +\left.\frac{1}{\sin \theta_{x}} P_{\ell}^{\prime}(\langle x, y\rangle)\left\{\sin \theta_{y} \cos \left(\varphi_{x}-\varphi_{y}\right)\right\}\right|_{x=y} \\
& =P_{\ell}^{\prime}(1) .
\end{aligned}
$$

Lemma 17. For all points $x=\left(\theta_{x}, \varphi_{x}\right) \in \mathbb{S}^{2} \backslash\{N, S\}$,

$$
\left.\mathbb{E}\left[\partial_{21 ; x} f_{\ell}(x) \partial_{22 ; y} f_{\ell}(y)\right]\right|_{x=y}=0 .
$$

Proof. We have that

$$
\begin{aligned}
& \left.\mathbb{E}\left[\partial_{21 ; x} f_{\ell}(x) \partial_{22 ; y} f_{\ell}(y)\right]\right|_{x=y} \\
& =\left.\frac{1}{\sin \theta_{x}} P_{\ell}^{\prime \prime \prime \prime}(\langle x, y\rangle)\left\{-\sin \theta_{x} \cos \theta_{y}+\cos \theta_{x} \sin \theta_{y} \cos \left(\varphi_{x}-\varphi_{y}\right)\right\}^{2}\left\{\sin ^{2} \theta_{x} \sin ^{2}\left(\varphi_{x}-\varphi_{y}\right)\right\}\right|_{x=y} \\
& +\left.\frac{1}{\sin \theta_{x}} P_{\ell}^{\prime \prime \prime}(\langle x, y\rangle)\left\{-\sin \theta_{x} \cos \theta_{y}+\cos \theta_{x} \sin \theta_{y} \sin \left(\varphi_{x}-\varphi_{y}\right)\right\}\left\{\sin ^{2} \theta_{x} \sin ^{2}\left(\varphi_{x}-\varphi_{y}\right)\right\}\right|_{x=y} \\
& +\left.\frac{1}{\sin \theta_{x}} P_{\ell}^{\prime \prime \prime}(\langle x, y\rangle)\left\{-\sin \theta_{x} \cos \theta_{y}+\cos \theta_{x} \sin \theta_{y} \cos \left(\varphi_{x}-\varphi_{y}\right)\right\}\left\{\sin ^{2} \theta_{x} 2 \sin \left(\varphi_{x}-\varphi_{y}\right) \cos \left(\varphi_{x}-\varphi_{y}\right)\right\}\right|_{x=y} \\
& +\left.\frac{1}{\sin \theta_{x}} P_{\ell}^{\prime \prime \prime}(\langle x, y\rangle)\left\{-\sin \theta_{x} \cos \theta_{y}+\cos \theta_{x} \sin \theta_{y} \cos \left(\varphi_{x}-\varphi_{y}\right)\right\}\left\{2 \sin \theta_{x} \cos \theta_{x} \sin ^{2}\left(\varphi_{x}-\varphi_{y}\right)\right\}\right|_{x=y} \\
& +\left.\frac{1}{\sin \theta_{x}} P_{\ell}^{\prime \prime}(\langle x, y\rangle)\left\{2 \sin \theta_{x} \cos \theta_{x} 2 \sin \left(\varphi_{x}-\varphi_{y}\right) \cos \left(\varphi_{x}-\varphi_{y}\right)\right\}\right|_{x=y} \\
& -\left.\frac{1}{\sin \theta_{x}} \frac{1}{\sin \theta_{y}} P_{\ell}^{\prime \prime \prime}(\langle x, y\rangle)\left\{-\sin \theta_{x} \cos \theta_{y}+\cos \theta_{x} \sin \theta_{y} \cos \left(\varphi_{x}-\varphi_{y}\right)\right\}^{2} \sin \theta_{x} \cos \left(\varphi_{x}-\varphi_{x}\right)\right|_{x=y} \\
& -\left.\frac{1}{\sin \theta_{x}} \frac{1}{\sin \theta_{y}} P_{\ell}^{\prime \prime}(\langle x, y\rangle)\left\{-\sin \theta_{x} \cos \theta_{y}-\cos \theta_{x} \sin \theta_{y} \sin \left(\varphi_{x}-\varphi_{y}\right)\right\} \sin \theta_{x} \cos \left(\varphi_{x}-\varphi_{x}\right)\right|_{x=y} \\
& +\left.\frac{1}{\sin \theta_{x}} \frac{1}{\sin \theta_{y}} P_{\ell}^{\prime \prime}(\langle x, y\rangle)\left\{-\sin \theta_{x} \cos \theta_{y}+\cos \theta_{x} \sin \theta_{y} \cos \left(\varphi_{x}-\varphi_{y}\right)\right\} \sin \theta_{x} \sin \left(\varphi_{x}-\varphi_{x}\right)\right|_{x=y} \\
& -\left.\frac{1}{\sin \theta_{x}} \frac{1}{\sin \theta_{y}} P_{\ell}^{\prime \prime}(\langle x, y\rangle)\left\{-\sin \theta_{x} \cos \theta_{y}+\cos \theta_{x} \sin \theta_{y} \cos \left(\varphi_{x}-\varphi_{y}\right)\right\} \cos \theta_{x} \cos \left(\varphi_{x}-\varphi_{x}\right)\right|_{x=y}
\end{aligned}
$$




$$
+\left.\frac{1}{\sin \theta_{x}} \frac{1}{\sin \theta_{y}} P_{\ell}^{\prime}(\langle x, y\rangle) \cos \theta_{x} \sin \left(\varphi_{x}-\varphi_{x}\right)\right|_{x=y},
$$

so that for all $x \in \mathbb{S}^{2}$,

as claimed.

$$
\left.\mathbb{E}\left[\partial_{21 ; x} T_{\ell}(x) \partial_{22 ; y} T_{\ell}(y)\right]\right|_{x=y}=0,
$$

The next variance is more delicate:

Lemma 18. For all points $x=\left(\theta_{x}, \varphi_{x}\right) \in \mathbb{S}^{2} \backslash\{N, S\}$,

$$
\left.\mathbb{E}\left[\partial_{22 ; x} f_{\ell}(x) \partial_{22 ; y} f_{\ell}(y)\right]\right|_{x=y}=3 P_{\ell}^{\prime \prime}(1)+P_{\ell}^{\prime}(1)+\cot ^{2} \theta_{x} P_{\ell}^{\prime}(1) .
$$

Proof. Here we obtain

$$
\begin{aligned}
& \left.\mathbb{E}\left[\partial_{22 ; x} f_{\ell}(x) \partial_{22 ; y} f_{\ell}(y)\right]\right|_{x=y} \\
& =\left.\partial_{22 ; x} \frac{1}{\sin ^{2} \theta_{y}} P_{\ell}^{\prime \prime}(\langle x, y\rangle)\left\{\sin ^{2} \theta_{x} \sin ^{2} \theta_{y} \sin ^{2}\left(\varphi_{x}-\varphi_{y}\right)\right\}\right|_{x=y} \\
& -\left.\partial_{22 ; x} \frac{1}{\sin ^{2} \theta_{y}} P_{\ell}^{\prime}(\langle x, y\rangle) \sin \theta_{x} \sin \theta_{y} \cos \left(\varphi_{x}-\varphi_{y}\right)\right|_{x=y} \\
& =A+B+C+D,
\end{aligned}
$$

for

$$
\begin{gathered}
A=\left.P_{\ell}^{\prime \prime \prime \prime}(\langle x, y\rangle)\left\{-\sin \theta_{y} \sin \left(\varphi_{x}-\varphi_{y}\right)\right\}^{2}\left\{\sin ^{2} \theta_{x} \sin ^{2}\left(\varphi_{x}-\varphi_{y}\right)\right\}\right|_{x=y} \\
+\left.\frac{1}{\sin \theta_{x}} P_{\ell}^{\prime \prime \prime}(\langle x, y\rangle)\left\{-\sin \theta_{y} \cos \left(\varphi_{x}-\varphi_{y}\right)\right\}\left\{\sin ^{2} \theta_{x} \sin ^{2}\left(\varphi_{x}-\varphi_{y}\right)\right\}\right|_{x=y} \\
+\left.\frac{1}{\sin \theta_{x}} P_{\ell}^{\prime \prime \prime}(\langle x, y\rangle)\left\{-\sin \theta_{y} \sin \left(\varphi_{x}-\varphi_{y}\right)\right\}\left\{2 \sin ^{2} \theta_{x} \sin \left(\varphi_{x}-\varphi_{y}\right) \cos \left(\varphi_{x}-\varphi_{y}\right)\right\}\right|_{x=y}=0 \\
B=\left.P_{\ell}^{\prime \prime \prime}(\langle x, y\rangle)\left\{-\sin \theta_{y} \sin \left(\varphi_{x}-\varphi_{y}\right)\right\}\left\{2 \sin \theta_{x} \sin \left(\varphi_{x}-\varphi_{y}\right) \cos \left(\varphi_{x}-\varphi_{y}\right)\right\}\right|_{x=y} \\
+\left.P_{\ell}^{\prime \prime}(\langle x, y\rangle)\left\{2 \cos \left(\varphi_{x}-\varphi_{y}\right) \cos \left(\varphi_{x}-\varphi_{y}\right)\right\}\right|_{x=y} \\
-\left.P_{\ell}^{\prime \prime}(\langle x, y\rangle)\left\{2 \sin \left(\varphi_{x}-\varphi_{y}\right) \sin \left(\varphi_{x}-\varphi_{y}\right)\right\}\right|_{x=y}=2 P_{\ell}^{\prime \prime}(1), \\
C=-\frac{1}{\sin \theta_{y}} P_{\ell}^{\prime \prime \prime}(\langle x, y\rangle)\left\{-\sin \theta_{y} \sin \left(\varphi_{x}-\varphi_{y}\right)\right\}^{2} \sin \theta_{x} \cos \left(\varphi_{x}-\varphi_{y}\right) \\
+\left.\frac{1}{\sin \theta_{y}} P_{\ell}^{\prime \prime}(\langle x, y\rangle)\left\{\sin \theta_{y} \cos \left(\varphi_{x}-\varphi_{y}\right)\right\} \cos \left(\varphi_{x}-\varphi_{y}\right)\right|_{x=y} \\
-\left.\frac{1}{\sin \theta_{y}} P_{\ell}^{\prime \prime}(\langle x, y\rangle)\left\{\sin \theta_{y} \sin \left(\varphi_{x}-\varphi_{y}\right)\right\} \sin \left(\varphi_{x}-\varphi_{y}\right)\right|_{x=y}=P_{\ell}^{\prime \prime}(1) \\
D=-\left.\frac{1}{\sin \theta_{y}} P_{\ell}^{\prime \prime}(\langle x, y\rangle) \sin \theta_{y} \sin \left(\varphi_{x}-\varphi_{y}\right) \sin \left(\varphi_{x}-\varphi_{y}\right)\right|_{x=y} \\
\quad+\left.\frac{1}{\sin \theta_{x}} \frac{1}{\sin \theta_{y}} P_{\ell}^{\prime}(\langle x, y\rangle) \cos \left(\varphi_{x}-\varphi_{y}\right)\right|_{x=y}=\frac{1}{\sin ^{2} \theta_{x}} P_{\ell}^{\prime}(1)
\end{gathered}
$$

Summing up, we obtain

$$
\left.\mathbb{E}\left[\partial_{22 ; x} T_{\ell}(x) \partial_{22 ; y} T_{\ell}(y)\right]\right|_{x=y}=3 P_{\ell}^{\prime \prime}(1)+\frac{1}{\sin ^{2} \theta_{x}} P_{\ell}^{\prime}(1)=3 P_{\ell}^{\prime \prime}(1)+P_{\ell}^{\prime}(1)+\cot ^{2} \theta_{x} P_{\ell}^{\prime}(1) .
$$

Lemma 19. For all points $x=\left(\theta_{x}, \varphi_{x}\right) \in \mathbb{S}^{2} \backslash\{N, S\}$,

$$
\left.\mathbb{E}\left[\partial_{11 ; x} f_{\ell}(x) \partial_{21 ; y} f_{\ell}(y)\right]\right|_{x=y}=0 .
$$

Proof. The result can be established by similar computations to those performed in the other Lemmas; alternatively, in this case it follows immediately by noting that $\left\{\partial_{11} f_{\ell}(x)\right\},\left\{\partial_{21 ; y} f_{\ell}(x)\right\}$ are the partial derivatives $\partial_{1}, \partial_{2}$ of the constant variance field $\partial_{1} f_{\ell}$.

Our final lemma is the following 
Lemma 20. For all points $x=\left(\theta_{x}, \varphi_{x}\right) \in \mathbb{S}^{2} \backslash\{N, S\}$,

$$
\left.\mathbb{E}\left[\partial_{21 ; x} f_{\ell}(x) \partial_{21 ; y} f_{\ell}(y)\right]\right|_{x=y}=P_{\ell}^{\prime \prime}(1)+P_{\ell}^{\prime}(1) \frac{\cos ^{2} \theta_{x}}{\sin ^{2} \theta_{x}} .
$$

Proof.

$$
\begin{aligned}
& \left.\mathbb{E}\left[\partial_{21 ; x} f_{\ell}(x) \partial_{21 ; y} f_{\ell}(y)\right]\right|_{x=y} \\
& =\left.\partial_{21 ; x} \partial_{2 ; y}\left\{P_{\ell}^{\prime}(\langle x, y\rangle)\left\{-\cos \theta_{x} \sin \theta_{y}+\sin \theta_{x} \cos \theta_{y} \cos \left(\varphi_{x}-\varphi_{y}\right)\right\}\right\}\right|_{x=y} \\
& =\left.\partial_{21 ; x}\left\{P_{\ell}^{\prime \prime}(\langle x, y\rangle)\left\{\sin \theta_{x} \sin \left(\varphi_{x}-\varphi_{y}\right)\right\}\left\{-\cos \theta_{x} \sin \theta_{y}+\sin \theta_{x} \cos \theta_{y} \cos \left(\varphi_{x}-\varphi_{x}\right)\right\}\right\}\right|_{x=y} \\
& +\left.\partial_{21 ; x}\left\{P_{\ell}^{\prime}(\langle x, y\rangle)\left\{\frac{\sin \theta_{x}}{\sin \theta_{y}} \cos \theta_{y} \sin \left(\varphi_{x}-\varphi_{y}\right)\right\}\right\}\right|_{x=y} \\
& =A+B+C+D+E,
\end{aligned}
$$

where

$$
\begin{aligned}
& A=P_{\ell}^{\prime \prime \prime \prime}(\langle x, y\rangle)\left\{-\sin \theta_{y} \sin \left(\varphi_{x}-\varphi_{y}\right)\right\}\left\{-\sin \theta_{x} \cos \theta_{y}+\cos \theta_{x} \sin \theta_{y} \cos \left(\varphi_{x}-\varphi_{x}\right)\right\} \\
& \times\left.\left\{\sin \theta_{x} \sin \left(\varphi_{x}-\varphi_{y}\right)\right\}\left\{-\cos \theta_{x} \sin \theta_{y}+\sin \theta_{x} \cos \theta_{y} \cos \left(\varphi_{x}-\varphi_{x}\right)\right\}\right|_{x=y} \\
& +\left.\left\{P_{\ell}^{\prime \prime \prime}(\langle x, y\rangle)\left\{-\cos \theta_{x} \sin \theta_{y} \sin \left(\varphi_{x}-\varphi_{x}\right)\right\}\left\{\sin \theta_{x} \sin \left(\varphi_{x}-\varphi_{y}\right)\right\}\left\{-\cos \theta_{x} \sin \theta_{y}+\sin \theta_{x} \cos \theta_{y} \cos \left(\varphi_{x}-\varphi_{x}\right)\right\}\right\}\right|_{x=y} \\
& +\left\{P_{\ell}^{\prime \prime \prime}(\langle x, y\rangle)\left\{-\sin \theta_{x} \cos \theta_{y}+\cos \theta_{x} \sin \theta_{y} \cos \left(\varphi_{x}-\varphi_{x}\right)\right\}\left\{\sin \theta_{x} \cos \left(\varphi_{x}-\varphi_{y}\right)\right\}\right. \\
& \left.\times\left\{-\cos \theta_{x} \sin \theta_{y}+\sin \theta_{x} \cos \theta_{y} \cos \left(\varphi_{x}-\varphi_{x}\right)\right\}\right\}\left.\right|_{x=y} \\
& +\left.\left\{P_{\ell}^{\prime \prime \prime}(\langle x, y\rangle)\left\{-\sin \theta_{x} \cos \theta_{y}+\cos \theta_{x} \sin \theta_{y} \cos \left(\varphi_{x}-\varphi_{x}\right)\right\}\left\{\sin \theta_{x} \sin \left(\varphi_{x}-\varphi_{y}\right)\right\}\left\{-\cos \theta_{y} \sin \left(\varphi_{x}-\varphi_{x}\right)\right\}\right\}\right|_{x=y}=0 \text {, } \\
& B=\left.\partial_{2 ; x}\left\{P_{\ell}^{\prime \prime}(\langle x, y\rangle)\left\{\cos \theta_{x} \sin \left(\varphi_{x}-\varphi_{y}\right)\right\}\left\{-\cos \theta_{x} \sin \theta_{y}+\sin \theta_{x} \cos \theta_{y} \cos \left(\varphi_{x}-\varphi_{y}\right)\right\}\right\}\right|_{x=y} \\
& =\left.\left\{P_{\ell}^{\prime \prime \prime}(\langle x, y\rangle)\left\{-\sin \theta_{y} \sin \left(\varphi_{x}-\varphi_{x}\right)\right\}\left\{\cos \theta_{x} \sin \left(\varphi_{x}-\varphi_{y}\right)\right\}\left\{-\cos \theta_{x} \sin \theta_{y}+\sin \theta_{x} \cos \theta_{y} \cos \left(\varphi_{x}-\varphi_{y}\right)\right\}\right\}\right|_{x=y} \\
& +\left.\left\{P_{\ell}^{\prime \prime}(\langle x, y\rangle)\left\{\frac{\cos \theta_{x}}{\sin \theta_{x}} \cos \left(\varphi_{x}-\varphi_{y}\right)\right\}\left\{-\cos \theta_{x} \sin \theta_{y}+\sin \theta_{x} \cos \theta_{y} \cos \left(\varphi_{x}-\varphi_{y}\right)\right\}\right\}\right|_{x=y} \\
& +\left.\left\{P_{\ell}^{\prime \prime}(\langle x, y\rangle)\left\{\cos \theta_{x} \sin \left(\varphi_{x}-\varphi_{y}\right)\right\}\left\{\cos \theta_{y} \sin \left(\varphi_{x}-\varphi_{y}\right)\right\}\right\}\right|_{x=y}=0, \\
& C=\left.\partial_{2 ; x}\left\{P_{\ell}^{\prime \prime}(\langle x, y\rangle)\left\{\sin \theta_{x} \sin \left(\varphi_{x}-\varphi_{y}\right)\right\}\left\{\sin \theta_{x} \sin \theta_{y}+\cos \theta_{x} \cos \theta_{y} \cos \left(\varphi_{x}-\varphi_{x}\right)\right\}\right\}\right|_{x=y} \\
& =\left.\left\{P_{\ell}^{\prime \prime \prime}(\langle x, y\rangle)\left\{-\sin \theta_{y} \sin \left(\varphi_{x}-\varphi_{y}\right)\right\}\left\{\sin \theta_{x} \sin \left(\varphi_{x}-\varphi_{y}\right)\right\}\left\{\sin \theta_{x} \sin \theta_{y}+\cos \theta_{x} \cos \theta_{y} \cos \left(\varphi_{x}-\varphi_{x}\right)\right\}\right\}\right|_{x=y} \\
& +\left.P_{\ell}^{\prime \prime}(\langle x, y\rangle)\left\{\cos \left(\varphi_{x}-\varphi_{y}\right)\right\}\left\{\sin \theta_{x} \sin \theta_{y}+\cos \theta_{x} \cos \theta_{y} \cos \left(\varphi_{x}-\varphi_{x}\right)\right\}\right|_{x=y} \\
& +\left.\left\{P_{\ell}^{\prime \prime}(\langle x, y\rangle)\left\{\sin \left(\varphi_{x}-\varphi_{y}\right)\right\}\left\{-\cos \theta_{x} \cos \theta_{y} \sin \left(\varphi_{x}-\varphi_{x}\right)\right\}\right\}\right|_{x=y}=P_{\ell}^{\prime \prime}(1), \\
& D=\left.\partial_{2 ; x}\left\{P_{\ell}^{\prime \prime}(\langle x, y\rangle)\left\{\frac{\sin \theta_{x}}{\sin \theta_{y}} \cos \theta_{y} \sin \left(\varphi_{x}-\varphi_{y}\right)\right\}\left\{-\sin \theta_{x} \cos \theta_{y}+\cos \theta_{x} \sin \theta_{y} \cos \left(\varphi_{x}-\varphi_{x}\right)\right\}\right\}\right|_{x=y} \\
& =\left.\left\{P_{\ell}^{\prime \prime \prime}(\langle x, y\rangle)\left\{-\sin \theta_{y} \sin \left(\varphi_{x}-\varphi_{x}\right)\right\}\left\{\frac{\sin \theta_{x}}{\sin \theta_{y}} \cos \theta_{y} \sin \left(\varphi_{x}-\varphi_{y}\right)\right\}\left\{-\sin \theta_{x} \cos \theta_{y}+\cos \theta_{x} \sin \theta_{y} \cos \left(\varphi_{x}-\varphi_{x}\right)\right\}\right\}\right|_{x=y} \\
& +\left.\left\{P_{\ell}^{\prime \prime}(\langle x, y\rangle)\left\{\frac{1}{\sin \theta_{y}} \cos \theta_{y} \cos \left(\varphi_{x}-\varphi_{y}\right)\right\}\left\{-\sin \theta_{x} \cos \theta_{y}+\cos \theta_{x} \sin \theta_{y} \cos \left(\varphi_{x}-\varphi_{x}\right)\right\}\right\}\right|_{x=y} \\
& +\left.\left\{P_{\ell}^{\prime \prime}(\langle x, y\rangle)\left\{\frac{1}{\sin \theta_{y}} \cos \theta_{y} \sin \left(\varphi_{x}-\varphi_{y}\right)\right\} \frac{1}{\sin \theta_{x}}\left\{-\sin \theta_{x} \cos \theta_{y}+\cos \theta_{x} \sin \theta_{y} \sin \left(\varphi_{x}-\varphi_{x}\right)\right\}\right\}\right|_{x=y}=0,
\end{aligned}
$$

and finally

$$
\begin{aligned}
E & =\left.\partial_{2 ; x}\left\{P_{\ell}^{\prime}(\langle x, y\rangle)\left\{\frac{\cos \theta_{x}}{\sin \theta_{y}} \cos \theta_{y} \sin \left(\varphi_{x}-\varphi_{y}\right)\right\}\right\}\right|_{x=y} \\
& =\left.\left\{P_{\ell}^{\prime \prime}(\langle x, y\rangle)\left\{-\sin \theta_{y} \sin \left(\varphi_{x}-\varphi_{y}\right)\right\}\left\{\frac{\cos \theta_{x}}{\sin \theta_{y}} \cos \theta_{y} \sin \left(\varphi_{x}-\varphi_{y}\right)\right\}\right\}\right|_{x=y}
\end{aligned}
$$




$$
+\left.\left\{P_{\ell}^{\prime}(\langle x, y\rangle)\left\{\frac{\cos \theta_{x}}{\sin \theta_{y}} \frac{\cos \theta_{y}}{\sin \theta_{x}} \cos \left(\varphi_{x}-\varphi_{y}\right)\right\}\right\}\right|_{x=y}=P_{\ell}^{\prime}(1) \frac{\cos ^{2} \theta_{x}}{\sin ^{2} \theta_{x}}
$$

\section{Appendix C: The Conditional Expectation}

Lemma 21. For all $(x, y) \in \mathbb{S}^{2} \times \mathbb{S}^{2}, x \neq y$, we have that

$$
\mu_{\ell}(x, y)=O_{\ell}\left(d_{\mathbb{S}^{2}}(x, y)\right) .
$$

Proof. We need to compute the $6 \times 4$ matrix

$$
B_{\ell}^{T}(x, y) A_{\ell}^{-1}(x, y)
$$

where

$$
A_{\ell}(x, y)=\left(\begin{array}{cccc}
\frac{\lambda_{\ell}}{2} & 0 & \alpha_{1, \ell}(x, y) & 0 \\
0 & \frac{\lambda_{\ell}}{2} & 0 & \alpha_{2, \ell}(x, y) \\
\alpha_{1, \ell}(x, y) & 0 & \frac{\lambda_{\ell}}{2} & 0 \\
0 & \alpha_{2, \ell}(x, y) & 0 & \frac{\lambda_{\ell}}{2}
\end{array}\right) ;
$$

Let us start by computing

$$
A_{\ell}^{-1}(x, y)=\left(\begin{array}{cccc}
2 \frac{\lambda_{\ell}}{\lambda_{\ell}^{2}-4 \alpha_{1, \ell}^{2}(x, y)} & 0 & -\frac{4}{\lambda_{\ell}^{2}-4 \alpha_{1, \ell}^{2}(x, y)} \alpha_{1, \ell}(x, y) & 0 \\
0 & 2 \frac{\lambda_{\ell}}{\lambda_{\ell}^{2}-4 \alpha_{2, \ell}^{2}(x, y)} & 0 & -\frac{4}{\lambda_{\ell}^{2}-4 \alpha_{2, \ell}^{2}} \alpha_{2, \ell}(x, y) \\
-\frac{4}{\lambda_{\ell}^{2}-4 \alpha_{1, \ell}^{2}(x, y)} \alpha_{1, \ell}(x, y) & 0 & 2 \frac{\lambda_{\ell}}{\lambda_{\ell}^{2}-4 \alpha_{1, \ell}^{2}(x, y)} & 0 \\
0 & -\frac{4}{\lambda_{\ell}^{2}-4 \alpha_{2, \ell}^{2}(x, y)} \alpha_{2, \ell}(x, y) & 0 & 2 \frac{\lambda_{\ell}}{\lambda_{\ell}^{2}-4 \alpha_{2, \ell}^{2}(x, y)}
\end{array}\right) .
$$

Hence, omitting for brevity the arguments $(x, y)$

$$
\begin{aligned}
& B_{\ell}^{T} A_{\ell}^{-1}\left(\begin{array}{l}
u_{1} \\
u_{2} \\
u_{1} \\
u_{2}
\end{array}\right) \\
& =\left(\begin{array}{cccc}
0 & 0 & 0 & \beta_{2, \ell} \\
0 & 0 & \beta_{1, \ell} & 0 \\
0 & 0 & 0 & \beta_{3, \ell} \\
-\beta_{2, \ell} & 0 & 0 & 0 \\
0 & \beta_{1, \ell} & 0 & 0 \\
-\beta_{3, \ell} & 0 & 0 & 0
\end{array}\right)\left(\begin{array}{cccc}
2 \frac{\lambda_{\ell}}{\lambda_{\ell}^{2}-4 \alpha_{1, \ell}^{2}} & 0 & -\frac{4}{\lambda_{\ell}^{2}-4 \alpha_{1, \ell}^{2}} \alpha_{1, \ell} & 0 \\
0 & 2 \frac{\lambda_{\ell}}{\lambda_{\ell}^{2}-4 \alpha_{2, \ell}^{2}} & 0 & -\frac{4}{\lambda_{\ell}^{2}-4 \alpha_{2, \ell}^{2}} \alpha_{2, \ell} \\
-\frac{4}{\lambda_{\ell}^{2}-4 \alpha_{1, \ell}^{2}} \alpha_{1, \ell} & 0 & 2 \frac{\lambda_{\ell}}{\lambda_{\ell}^{2}-4 \alpha_{1, \ell}^{2}} & 0 \\
0 & -\frac{4}{\lambda_{\ell}^{2}-4 \alpha_{2, \ell}^{2}} \alpha_{2, \ell} & 0 & 2 \frac{\lambda_{\ell}}{\lambda_{\ell}^{2}-4 \alpha_{2, \ell}^{2}}
\end{array}\right)\left(\begin{array}{l}
u_{1} \\
u_{2} \\
u_{1} \\
u_{2}
\end{array}\right) \\
& =\left(\begin{array}{cccc}
0 & -4 \frac{\beta_{2, \ell}}{\lambda_{\ell}^{2}-4 \alpha_{2, \ell}^{2}} \alpha_{2, \ell} & 0 & 2 \beta_{2, \ell} \frac{\lambda_{\ell}}{\lambda_{\ell}^{2}-4 \alpha_{2, \ell}^{2}} \\
-4 \frac{\beta_{1, \ell}}{\lambda_{\ell}^{2}-4 \alpha_{1, \ell}^{2}} \alpha_{1, \ell} & 0 & 2 \beta_{1, \ell} \frac{\lambda_{\ell}}{\lambda_{\ell}^{2}-4 \alpha_{1, \ell}^{2}} & 0 \\
0 & -4 \frac{\beta_{3, \ell}}{\lambda_{\ell}^{2}-4 \alpha_{2, \ell}^{2}} \alpha_{2, \ell} & 0 & 2 \beta_{3, \ell} \frac{\lambda_{\ell}}{\lambda_{\ell}^{2}-4 \alpha_{2, \ell}^{2}} \\
-2 \beta_{2, \ell \frac{\lambda_{\ell}}{\lambda_{\ell}^{2}-4 \alpha_{1, \ell}^{2}}} & 0 & 4 \frac{\beta_{2, \ell}}{\lambda_{\ell}^{2}-4 \alpha_{1, \ell}^{2}} \alpha_{1, \ell} & 0 \\
0 & 2 \beta_{1, \ell} \frac{\lambda_{\ell}}{\lambda_{\ell}^{2}-4 \alpha_{2, \ell}^{2}} & 0 & -4 \frac{\beta_{1, \ell}}{\lambda_{\ell}^{2}-4 \alpha_{2, \ell}^{2}} \alpha_{2, \ell} \\
-2 \beta_{3, \ell} \frac{\lambda_{\ell}}{\lambda_{\ell}^{2}-4 \alpha_{1, \ell}^{2}} & 0 & 4 \frac{\beta_{3, \ell}}{\lambda_{\ell}^{2}-4 \alpha_{1, \ell}^{2}} \alpha_{1, \ell} & 0
\end{array}\right)\left(\begin{array}{l}
u_{1} \\
u_{2} \\
u_{1} \\
u_{2}
\end{array}\right) \\
& =\left(\begin{array}{c}
\frac{2 \beta_{2, \ell}}{\lambda_{\ell}^{2}-4 \alpha_{2, \ell}^{2}}\left(\lambda_{\ell} u_{2}-2 \alpha_{2, \ell} u_{2}\right) \\
\frac{2 \beta_{1, \ell}}{\lambda_{\ell}^{2}-4 \alpha_{1, \ell}^{2}}\left(\lambda_{\ell} u_{1}-2 \alpha_{2, \ell} u_{1}\right) \\
\frac{2 \beta_{3, \ell}}{\lambda_{\ell}^{2}-4 \alpha_{2, \ell}^{2}}\left(\lambda_{\ell} u_{2}-2 \alpha_{2, \ell} u_{2}\right) \\
-\frac{2 \beta_{2, \ell}}{\lambda_{\ell}^{2}-4 \alpha_{1, \ell}^{2}}\left(\lambda_{\ell} u_{1}-2 \alpha_{2, \ell} u_{1}\right) \\
\frac{2 \beta_{1, \ell}}{\lambda_{\ell}^{2}-4 \alpha_{2, \ell}^{2}}\left(\lambda_{\ell} u_{2}-2 \alpha_{2, \ell} u_{2}\right) \\
-\frac{2 \beta_{3, \ell}}{\lambda_{\ell}^{2}-4 \alpha_{1, \ell}^{2}}\left(\lambda_{\ell} u_{1}-2 \alpha_{2, \ell} u_{1}\right)
\end{array}\right)=\left(\begin{array}{c}
\frac{2 \beta_{2, \ell} u_{2}}{\lambda_{\ell}+2 \alpha_{2, \ell}} \\
\frac{2 \beta_{1, \ell} u_{1}}{\lambda_{\ell}+2 \alpha_{1, \ell}} \\
\frac{2 \beta_{3, \ell} u_{2}}{\lambda_{\ell}+2 \alpha_{2, \ell}} \\
-\frac{2 \beta_{2, \ell} u_{1}}{\lambda_{\ell}+2 \alpha_{1, \ell}} \\
\frac{2 \beta_{1, \ell} u_{2}}{\lambda_{\ell}+2 \alpha_{2, \ell}} \\
-\frac{2 \beta_{3, \ell} u_{1}}{\lambda_{\ell}+2 \alpha_{1, \ell}}
\end{array}\right)=O_{\ell}(\varepsilon \sin \phi) .
\end{aligned}
$$


Let us now focus without loss of generality on "equator" points $x=\left(\frac{\pi}{2}, \varphi_{x}\right), y=\left(\frac{\pi}{2}, \varphi_{y}\right)$, and write $\phi:=$ $d_{\mathbb{S}^{2}}(x, y)=\left|\varphi_{x}-\varphi_{y}\right|$. It has been shown in [7] that the following expressions hold,

$$
\begin{gathered}
\beta_{1, \ell}(\phi)=\sin \phi P_{\ell}^{\prime \prime}(\cos \phi), \\
\beta_{2, \ell}(\phi)=\sin \phi \cos \phi P_{\ell}^{\prime \prime}(\cos \phi)+\sin \phi P_{\ell}^{\prime}(\cos \phi), \\
\beta_{3, \ell}(\phi)=-\sin ^{3} \phi P_{\ell}^{\prime \prime \prime}(\cos \phi)+3 \sin \phi \cos \phi P_{\ell}^{\prime \prime}(\cos \phi)+\sin \phi P_{\ell}^{\prime}(\cos \phi),
\end{gathered}
$$

whence

$$
\beta_{1, \ell}(\phi), \beta_{2, \ell}(\phi), \beta_{3, \ell}(\phi)=O_{\ell}(\sin \phi)=O_{\ell}(\phi), \text { as } \phi \rightarrow 0 .
$$

Recall also that

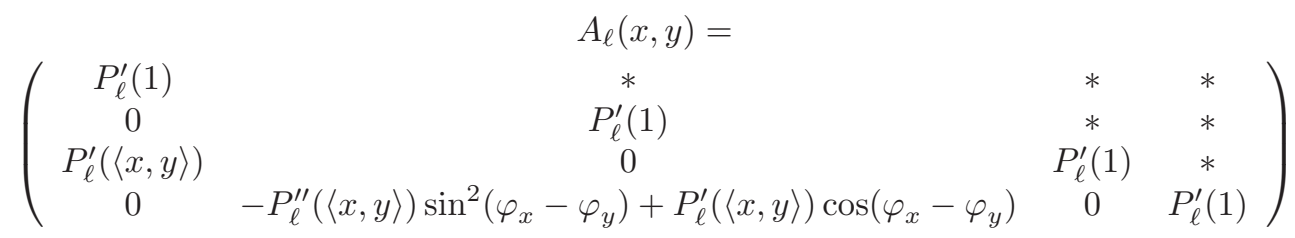

whence

$$
\begin{aligned}
& \alpha_{1, \ell}(x, y)=P_{\ell}^{\prime}(\langle x, y\rangle), \\
& \alpha_{2, \ell}(x, y)=-P_{\ell}^{\prime \prime}(\langle x, y\rangle) \sin ^{2}\left(\varphi_{x}-\varphi_{y}\right)+P_{\ell}^{\prime}(\langle x, y\rangle) \cos \left(\varphi_{x}-\varphi_{y}\right),
\end{aligned}
$$

and thus on the equator $\varphi_{x}=\varphi_{y}$

$$
\alpha_{1, \ell}(x, y)=P_{\ell}^{\prime}(\langle x, y\rangle)=\alpha_{2, \ell}(x, y)
$$

It hence follows that

$$
\begin{aligned}
\left|\mu_{i, \ell}(x, y)\right| & \leq \text { const }_{\ell} \times\left\{\left|u_{1}\right|+\left|u_{2}\right|\right\} \sin \phi \\
& \leq \text { const }_{\ell} \times\left\{\left|u_{1}\right|+\left|u_{2}\right|\right\} \times d_{\mathbb{S}^{2}}(x, y),
\end{aligned}
$$

where the constant can depend on $\left\{\lambda_{\ell}+2 \alpha_{1, \ell}\right\}^{-1}$, which is bounded because it is the inverse of a non-vanishing polynomial in a compact space. Hence the result is established.

Acknowledgements We are grateful to Maurizia Rossi and Igor Wigman for many insightful discussions. DM acknowledges the MIUR Excellence Department Project awarded to the Department of Mathematics, University of Rome Tor Vergata, CUP E83C18000100006.

Valentina Cammarota

Dipartimento di Scienze Statistiche

Sapienza Università di Roma

Piazzale Aldo Moro, 5

00185 Roma

email: valentina.cammarota@uniroma1.it

Domenico Marinucci

Dipartimento di Matematica

Università di Roma Tor Vergata

Via della Ricerca Scientifica, 1

00133 Roma

email: marinucc@mat.uniroma2.it 